\title{
Reliable Effects Screening: A Distributed Continuous Quality Assurance Process for Monitoring Performance Degradation in Evolving Software Systems
}

\author{
Cemal Yilmaz, Member, IEEE Computer Society, Adam Porter, Senior Member, IEEE, \\ Arvind S. Krishna, Atif M. Memon, Member, IEEE Computer Society, Douglas C. Schmidt, \\ Aniruddha S. Gokhale, and Balachandran Natarajan
}

\begin{abstract}
Developers of highly configurable performance-intensive software systems often use in-house performance-oriented "regression testing" to ensure that their modifications do not adversely affect their software's performance across its large configuration space. Unfortunately, time and resource constraints can limit in-house testing to a relatively small number of possible configurations, followed by unreliable extrapolation from these results to the entire configuration space. As a result, many performance bottlenecks escape detection until systems are fielded. In our earlier work, we improved the situation outlined above by developing an initial quality assurance process called "main effects screening." This process 1) executes formally designed experiments to identify an appropriate subset of configurations on which to base the performance-oriented regression testing, 2) executes benchmarks on this subset whenever the software changes, and 3) provides tool support for executing these actions on in-the-field and in-house computing resources. Our initial process had several limitations, however, since it was manually configured (which was tedious and error-prone) and relied on strong and untested assumptions for its accuracy (which made its use unacceptably risky in practice). This paper presents a new quality assurance process called "reliable effects screening" that provides three significant improvements to our earlier work. First, it allows developers to economically verify key assumptions during process execution. Second, it integrates several modeldriven engineering tools to make process configuration and execution much easier and less error prone. Third, we evaluate this process via several feasibility studies of three large, widely used performance-intensive software frameworks. Our results indicate that reliable effects screening can detect performance degradation in large-scale systems more reliably and with significantly less resources than conventional techniques.
\end{abstract}

Index Terms-Distributed continuous quality assurance, performance-oriented regression testing, design-of-experiments theory.

\section{INTRODUCTION}

THE quality of service (QoS) of many performanceintensive systems, such as scientific computing systems and distributed real-time and embedded (DRE) systems, depends heavily on various environmental factors. Example dependencies include the specific hardware and operating system on which systems run, installed versions of middleware and system library implementations, available language processing tools, specific software features that

- C. Yilmaz is with the IMB T.J. Watson Research Center, 19 Skyline Dr., Hawthorne, NY 10532. E-mail: cyilmaz@us.ibm.com.

- A. Porter and A.M. Memon are with the Department of Computer Science, University of Maryland, College Park, MD 20742.

E-mail: \{aporter, atif\}@cs.umd.edu.

- A.S. Krishna, D.C. Schmidt, A.S. Gokhale, and B. Natarajan are with the Department of Electrical Engineering and Computer Science, Vanderbilt University, Nashoille, TN 37325.

E-mail:arvindkr@qualcomm.com,schmidt@dre.vanderbilt.edu, a.gokhale@vanderbilt.edu.

- B. Natarajan is with Symantec, ?MAILING ADDRESS?.

E-mail: bala_natrajan@symantec.com.

Manuscript received 14 Dec. 2005; revised 26 July 2006; accepted 13 Nov. 2006; published online 28 Dec. 2006.

Recommended for acceptance by B. Littlewood.

For information on obtaining reprints of this article, please send e-mail to: tse@computer.org, and reference IEEECS Log Number TSE-0331-1205. are enabled/disabled for a given customer, and dynamic workload characteristics. Many of these dependencies are not known until deployment and some change frequently during a system's lifetime.

To accommodate these dependencies, users often need to tune infrastructure and software applications by (re)adjusting many (i.e., dozens to hundreds) of compile-time and runtime configuration options that record and control variable software parameters. These options are exposed at multiple system layers, including compiler flags and operating system, middleware, and application feature sets and runtime optimization settings. For example, there are $\sim 50$ configuration options for SQL Server 7.0, $\sim 200$ initialization parameters for Oracle 9 , and $\sim 90$ core configuration options for Apache HTTP Server Version 1.3. Although designing performance-intensive systems to include such configuration options promotes code reuse, enhances portability, and helps end users improve their QoS, it also yields an enormous family of "instantiated" systems, each of which might behave differently and, thus, may need quality assurance (QA). The size of these system families creates serious and often under-appreciated challenges for software developers, who must ensure that their 
decisions, additions, and modifications work across this large (and often dynamically changing) space. For example, consider that:

- Option settings that maximize performance for a particular environment may be ill-suited for different ones. Failures can and do manifest in some configurations, but not in others. Similarly, individual code changes can have different runtime effects in different configurations.

- Individual developers, especially those in smaller companies or in open-source projects, may not have access to the full range of hardware platforms, operating systems, middleware, class library versions, etc. over which the system must run. In these situations, individual QA efforts will necessarily be incomplete.

- Limited budgets, aggressive schedules, and rapidly changing code bases mean that QA efforts frequently focus on a relatively small number of system configurations, often chosen in an ad hoc fashion. Developers then unreliably extrapolate from this data to the entire configuration space, which allows quality problems to escape detection until systems are fielded.

In summary, resource constraints and the large number of possible system configurations make exhaustive evaluation infeasible for performance-intensive systems. Developers therefore need 1) ways to identify a small core set of configurations whose QA results can be reliably generalized across all configurations and 2) support for executing QA activities across a sufficiently rich and diverse set of computing platforms. To address these needs, our research [33], [31], [20], [32] has focused on system support and algorithms for distributed continuous quality assurance (DCQA) processes. DCQA helps improve software quality iteratively, opportunistically, and efficiently by executing QA tasks continuously across a grid of computing resources provided by end-users and developer communities.

In prior work [20], we created a prototype DCQA support environment called Skoll that helps developers create, execute, and analyze their own DCQA processes, as described in Section 2. To facilitate DCQA process development and validation, we also developed model-driven engineering tools for use with Skoll. We then used Skoll to design and execute an initial DCQA process, called "main effects screening" [33], whose goal was to estimate performance efficiently across all system configurations (hereafter called the "configuration space").

Main effects screening borrows ideas from statistical quality improvement techniques that have been applied widely in engineering and manufacturing, such as Exploratory Data Analysis [27], Robust Parameter Design [30], and Statistical Quality Control [23]. A central activity of these techniques is to identify aspects of a system or process that contribute substantially to outcome variation. We use similar ideas to identify important configuration options whose settings define the distribution of performance across all configurations by causing the majority of performance variation. Evaluating all combinations of these important options (and randomizing the other options), thus provides an inexpensive, but reliable estimate of performance across the entire configuration space.

Although our initial work on main effects screening presented in [33] showed promise, it also had several limitations. For example, the definition and execution of the process had many manual steps. To improve this, we have extended and better integrated several model-driven engineering (MDE) [25] tools, including the Options Configuration Modeling Language (OCML) [28], which models configuration options and interoption constraints, and the Benchmark Generation Modeling Language (BGML) [13], which models the QA tasks that observe and measure QoS behavior under different configurations and workloads. These MDE tools precisely capture common and variable parts of DCQA processes and the software systems to which they are applied. They also help reduce development and QA effort by generating configuration files and many other supporting code artifacts [2] needed to manage and control process execution across heterogeneous computing resources.

Another limitation with our initial main effects screening process was its dependence on strong and untested assumptions regarding the absence of interactions among certain groups of options. If these assumptions do not hold in practice, our results could be wildly incorrect. Moreover, we had no way to assess the validity of the assumptions without resorting to exhaustive testing, the avoidance of which motivated our DCQA process in the first place.

To remedy these problems, this paper describes further enhancements to our earlier work that significantly broaden its applicability with little additional operating cost. Our new DCQA process, called "reliable effects screening" is implemented using Skoll and its MDE tools, and relies on design-of-experiments (DOE) techniques called "screening designs" [30] and "D-optimal designs" [22]. Our reliable effects screening process first identifies a small subset of the most important performance-related configuration options by creating formally designed experiments and executing them across the Skoll grid. Whenever software changes occur thereafter, reliable effects screening then uses a far smaller amount of computing resources to estimate system performance across the entire configuration space by exhaustively exploring all combinations of the important options, while randomizing the rest. This subsequent analysis can even be run completely in-house, assuming appropriate computing platforms are available, since the reduced configuration space is much smaller than the original and thus more tractable using only in-house resources.

In addition to describing our new reliable effects screening DCQA process, this paper also evaluates this process empirically on ACE, TAO, and CIAO (dre.vanderbilt.edu), which are three widely used, production-quality, performance-intensive software frameworks. This evaluation indicates that 1 ) our reliable effects screening process can correctly and reliably identify the subset of options that are most important to system performance, 2) monitoring only these selected options helps to detect performance degradation quickly with an acceptable level of effort, and 3) alternative strategies with equivalent effort yield less reliable results. These results support our contention that 
reliable effects screening can inexpensively and quickly alert performance-intensive system developers to changes that degrade QoS, as well as provide them with much greater insight into their software's performance characteristics. The remainder of this paper is organized as follows: Section 2 summarizes the Skoll DCQA environment, Section 3 describes how we extended Skoll to implement the new reliable effect screening DCQA process to conduct performance-oriented regression testing efficiently, Section 4 presents the design and results of a feasibility study using ACE, TAO, and CIAO, Section 5 further discusses our empirical results and evaluates threats to the validity of our experiments, Section 6 presents guidelines on how to use reliable effects screening, Section 7 compares our research on reliable effect screening with related work, and Section 8 presents concluding remarks and outlines future directions of our DCQA process and tool research.

\section{An Overview of the SKoll DCQA ENVIRONMENT}

To improve the quality of performance-intensive systems across large configuration spaces, our work focuses on distributed continuous quality assurance (DCQA) processes [20] that evaluate various software qualities, such as portability, performance characteristics, and functional correctness "around-the-world and around-the-clock." ${ }^{11}$ To support this methodology, we developed Skoll, which is a model-driven engineering (MDE)-based DCQA environment (www.cs.umd.edu/projects/skoll). Skoll divides QA processes into multiple tasks, each of which is implemented as a generic process parametrized by one of several alternative configurations expressed via MDE tools. Example tasks might include running regression tests in one of many system configurations, evaluating system response time under one of several different input workloads, or measuring code execution coverage using one of several instrumentation schemes.

As shown in Fig. 1, these tasks are then intelligently and continuously distributed to-and executed by-clients across a grid of computing resources contributed by enduser and developer communities. The results of individual tasks are returned to servers at central collection sites, where they are merged and analyzed to steer subsequent iterations and ultimately to complete the overall QA process.

This section summarizes Skoll's key components and services, which include MDE tools for modeling system configurations and their constraints, a domain-specific modeling language (DSML) to describe these models, algorithms for scheduling and remotely executing tasks via planning technology that analyzes task results and adapts the DCQA process in real time, a DSML to package the subtasks, and techniques to interpret and visualize the results.

QA task space. Performance-intensive systems, such as the ACE+TAO+CIAO QoS-enabled middleware, provide a range (i.e., $\sim 500$ ) of configuration options that can be used

1. Naturally, DCQA processes can also be executed effectively in more constrained and smaller-scale environments, such as companywide intranets.

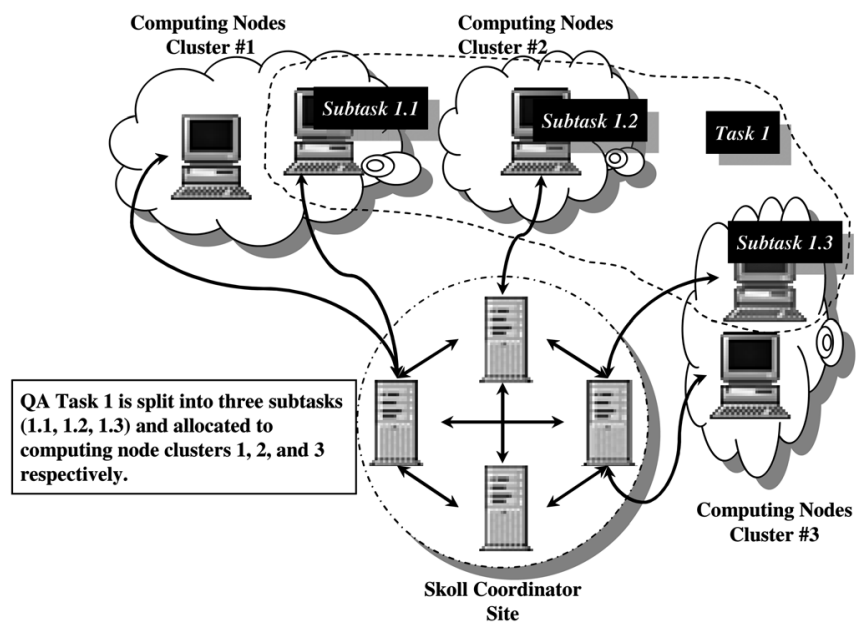

Fig. 1. The Skoll distributed continuous quality assurance architecture.

to tune its behavior. ${ }^{2}$ To be effective, DCQA processes must keep track of these options, in addition to other environmental information, such as OS platform, build tools used, and desired version numbers. This information is used to parameterize generic QA tasks and aids in planning the global QA process, e.g., by adapting the process dynamically and helping interpret the results.

In Skoll, tasks are generic processes parameterized by QA task options. These options capture information that is 1) varied under QA process control or 2) needed by the software to build and execute properly. These options are generally application-specific, including workload parameters, operating system, library implementations, compiler flags, or runtime optimization controls. Each option must take its value from a discrete number of settings. For example, in other work, our QA task model included a configuration option called OperatingSystem so Skoll could select appropriate binaries and build code for specific tasks [32].

QA task modeling. The QA task model underlies the DCQA process. Our experience [20], [13], [31] with the initial Skoll prototype taught us that building these models manually was tedious and error-prone. We therefore developed and integrated into Skoll the Options Configuration Modeling Language (OCML) [28]. OCML is an MDE tool that provides a DSML for modeling software configurations. For example, OCML defines a numeric option type for middleware options that can have numeric values, e.g., cache, buffer, or thread pool sizes. OCML is built atop the Generic Modeling Environment (GME) [16], which provides a metaprogrammable framework for creating DSMLs and generative tools via metamodels and model interpreters. For the feasibility study in Section 4, we used the OCML MDE tool to define the configuration model visually and generate the low-level formats used by other Skoll components.

Exploring the QA task space. Since the QA task spaces of many systems can be enormous, Skoll contains an Intelligent Steering Agent (ISA) [20] that uses AI planning techniques [21] to distribute QA tasks on available Skoll

2. All the ACE+TAO+CIAO's configuration options are described at www.dre.vanderbilt.edu/ schmidt/DOC_ROOT/TAO/docs/ Options.html. 
clients. When clients become available, they send a message to the Skoll server. Skoll's ISA then decides which task to assign it by considering many factors, including 1) the $\mathrm{QA}$ task model, which characterizes the subtasks that can be assigned legally, 2) the results of previous tasks, which capture what tasks have already been done and whether the results were successful, 3) global process goals, such as testing popular configurations more than rarely used ones or testing recently changed features more heavily than unchanged features, and (4) client characteristics and preferences, e.g., the selected configuration must be compatible with the OS running on the client machine or users can specify preferences that configurations must run with user-levelrather than superuser-level-protection modes.

After a valid configuration is chosen, the ISA packages the corresponding QA task into a job configuration, which consists of the code artifacts, configuration parameters, build instructions, and QA-specific code (e.g., developersupplied regression/performance tests) associated with a software project. Each job configuration is then sent to a Skoll client, which executes the job configuration and returns the results to the ISA. By default, the ISA simply stores these results.

In some experiments, however, we want to learn from incoming results. For example, when some configurations prove faulty, it makes no sense to retest them. Instead, we should refocus resources on other unexplored parts of the QA task space. When such dynamic behavior is desired, DCQA process designers develop customized adaptation strategies that Skoll uses to monitor the global process state, analyze it, and modify future task assignments in ways that improve process performance. One example of an adaptation strategy is the nearest neighbor search strategy, which allows a process to target failing QA task subspaces by preferentially testing the "neighbors" of a failing configuration, (i.e., other similar configurations that differ in one configuration option value) to see if they also fail [20].

Packaging QA tasks. With the initial Skoll prototype, developers who wanted to evaluate QoS issues had to provide handwritten benchmark programs. For example, $\mathrm{ACE}+\mathrm{TAO}+\mathrm{CIAO}$ developers creating such benchmarks to measure latency and throughput for a particular workload had to write 1) the header files and source code that implement the measurements, 2) the configuration and script files that tune the underlying Object Request Broker (ORB) and automate running tests and output generation, and 3) project build files (e.g., makefiles) required to generate executable binaries from source code. Our initial feasibility study [20] revealed that this process was tedious and error-prone.

To address these problems, we developed the Benchmark Generation Modeling Language (BGML) [14], which is an MDE tool that automates key QoS evaluation concerns of QoS-enabled middleware and applications, such as 1) modeling how distributed system components interact with each other and 2) representing metrics that can be applied to specific configuration options and platforms. Middleware/application developers can use BGML to graphically model interaction scenarios of interest. BGML automates the task of writing repetitive source code to

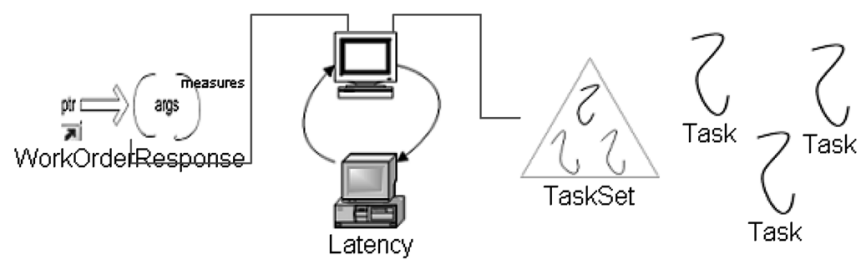

Fig. 2. Associating QoS with an operation in BGML.

perform benchmark experiments and generates syntactically and semantically valid source and benchmarking code.

Fig. 2 depicts how QA engineers can visually configure an experiment that measures end-to-end latency. As shown in Fig. 2, the latency metric was associated with an operation (WorkOrderResponse()) using BGML. BGML's Taskset element was also used to create background tasks that invoked the WorkOrderResponse() operation continuously for a fixed number of iterations.

Analysis of results. Since DCQA processes can be complex, Skoll users often need help to visualize, interpret, and leverage process results. Skoll therefore supports a variety of pluggable analysis tools, such as Classification Tree Analysis (CTA) [4]. In previous work [20], [31], we used CTA to diagnose options and settings that were the likely causes of specific test failures. For the work presented in this paper, we developed statistical tools to analyze data generated by the formally designed experiments described next.

\section{Performance-Oriented Regression Testing}

As software systems evolve, developers often run regression tests to detect unintended functional side effects. Developers of performance-intensive systems must also detect unintended side effects on end-to-end QoS. A common way to detect these effects is to run benchmarks when the system changes. As described in Section 1, however, these efforts can be confounded for systems with many possible configurations because time and resource constraints (and often high change frequencies) severely limit the number of configurations that can be examined using only in-house resources.

For example, our earlier experiences applying Skoll to ACE+TAO [20] showed that ACE+TAO developers have a limited view of their software's QoS since they routinely benchmark only a small number of common configurations. QoS degradations not readily seen in these configurations, therefore, can and do escape detection until systems based on ACE+TAO are fielded by end-users [20], [13]. The key problem here is that the ACE+TAO developers are benchmarking a small and unrepresentative sample of system configurations, so their extrapolations from this data are bound to be unreliable.

To address this problem, we have developed and evaluated the reliable effects screening process, which uses "design of experiments" theory [11] to determine an appropriate subset of system configurations to benchmark when the system changes. This section describes how we implemented the reliable effects screening process and 
applied it to ACE+TAO+CIAO and discusses several process choices developers must make when applying it.

\subsection{The Reliable Effects Screening Process}

Reliable effects screening (RES) is a process we developed to detect performance degradation rapidly across a large configuration space as a system changes. This process identifies a small subset of "important" configuration options that substantially affect variation in performance. Benchmarking a "screening suite" containing all combinations of these important option settings (with other options assigned randomly) should therefore provide a reliable estimate of performance across the entire configuration space at a fraction of the cost and effort of exhaustive benchmarking.

At a high level, the process involves the following steps:

1. Compute a formal experimental design based on the system's QA task model.

2. Execute that experimental design across volunteered computing resources in the Skoll computing grid by running and measuring benchmarks on specific configurations dictated by the experimental design devised in Step 1.

3. Collect, analyze, and display the data so that developers can identify the most important options, i.e., the options that affect performance most significantly.

4. Conduct supplementary analysis again on volunteered computing resources to check the basic assumptions underlying Step 1 and to confirm the results of Step 3.

5. Estimate overall performance (in-house, if possible) whenever the software changes by evaluating all combinations of the important options (while randomizing all other options).

6. Frequently recalibrate the important options by restarting the process since these effects can change over time, depending on how rapidly the subject system changes.

\subsection{Screening Designs Background}

The first step of reliable effects screening is to identify options accounting for the most performance variation across the system's QA task space. We do this by executing and analyzing formally designed experiments, called screening designs, which are described in Kolarik [11], Wu and Hamada [30], or the NIST Engineering Statistics Handbook (www.itl.nist.gov/div898/handbook/index.htm). Screening designs are highly economical plans for identifying important low-order effects, i.e., first-order, second-order, or third-order effects (where an $n$ th-order effect is an effect caused by the simultaneous interaction of $n$ factors).

To better understand screening designs, consider a full factorial (i.e., exhaustive) experimental design involving $k$ independent binary factors. The design's run size (number of experimental observations) is, therefore, $2^{k}$. Although such designs allow all first-order through $k$ th-order effects to be computed, they quickly become computationally expensive to run. Screening designs, in contrast, reduce costs by observing only a carefully selected subset of a full factorial design. The trade-off is that they cannot compute most higher-order effects because the selection of observations
TABLE 1 $2_{I V}^{4-1}$ Design (Binary Option Settings Are Encoded as $(-)$ or $\left.(+)\right)$

\begin{tabular}{|cccc|}
\hline $\mathrm{A}$ & $\mathrm{B}$ & $\mathrm{C}$ & $\mathrm{D}$ \\
\hline \hline- & - & - & - \\
+ & - & - & + \\
- & + & - & + \\
+ & + & - & - \\
- & - & + & + \\
+ & - & + & - \\
- & + & + & - \\
+ & + & + & + \\
\hline
\end{tabular}

aliases the effects of some lower-order interactions with some higher-order ones, i.e., it conflates certain high-order and low-order effects.

Which effects are conflated depends on the design's resolution. In resolution $R$ designs, no effects involving $i$ factors are aliased with effects involving less than $R-i$ factors. For instance, a resolution III design is useful to evaluate "clear" (no two aliased together) first-order effects, where all higher effects are negligible. Here all first-order effects will be clear, but they may be aliased with secondorder or higher-order effects. This conflation is justified only if the high-order effects are indeed negligible. If these assumptions are patently unreasonable, then a higher resolution may be needed.

In practice, statistical packages are used to compute specific screening designs. We used the SAS/QC [1] package in this work, but many other packages, such as MINITAB and SPSS, are also applicable. These packages will produce a screening design, assuming one can be found, given the following application-specific information: 1) a list of options and their settings, 2) a maximum run size, and 3) the design's resolution.

Since we already build our QA task model graphically using Skoll's MDE tools (see Section 2), we can just use a translator to convert it into the list of options and settings expected by our statistical package. The second and third items are intertwined and must be chosen by developers. In particular, higher resolution designs will yield more accurate estimates (assuming some higher-level effects exist), but require more observations. It is also often advisable to run more than the minimum number of observations needed for a given resolution to improve precision or to deal with noisy processes. Developers must balance these competing forces.

\subsection{Computing a Screening Design}

To demonstrate these choices, consider a hypothetical software system with four independent binary configuration options, $A$ through $D$, each with binary settings + and -. A full factorial design for this system involves $16\left(2^{4}\right)$ observations. We assume that our developers can only afford to gather eight observations. With so few observations, there is no design with clear first-order and secondorder effects. Developers must therefore either allow more observations or limit themselves to capturing only the four first-order effects, i.e., the effect of each option by itself. We assume they choose to stay with a run size of eight and to use a resolution IV design.

Given these choices, the developers generate one acceptable design using a statistical package. The design (which appears in Table 1) is identified uniquely as a $2_{I V}^{4-1}$ design, which means that the total number of options is 
four, that they will observe a $1 / 2\left(2^{-1}=1 / 2\right)$ fraction of the full factorial design, and that the design is a resolution IV screening design.

The design tool also outputs the aliasing structure $D=A B C$. We can see this aliasing in Table 1 , where the setting of option $D$ is the product of the settings of options $A, B$, and $C$ (think of + as 1 and - as -1 ). This dependence explains why the effect of option $D$ cannot be untangled from the interaction effect of options $A, B$, and $C$.

\subsection{Executing and Analyzing Screening Designs}

After defining the screening design, developers will execute it across the computing resources comprising the Skoll grid. In our later feasibility studies, each experimental observation involves measuring a developer-supplied benchmark program while the system runs in a particular configuration. Our QA engineers use BGML to generate workload and benchmark code. Once the data is collected, we analyze it to calculate the effects. Since our screening designs are balanced and orthogonal by construction (i.e., no bias in the observed data), the effect calculations are simple. For binary options (with settings - or + ), the effect of option $A$, $M E(A)$, is

$$
M E(A)=z(A-)-z(A+),
$$

where $z(A-)$ and $z(A+)$ are the mean values of the observed data over all runs where option $A$ is $(-)$ and where option $A$ is $(+)$, respectively. If required, secondorder effects can be calculated in a similar way. The interaction effect of options $A$ and $B, \operatorname{INT}(A, B)$ is:

$$
\begin{aligned}
\operatorname{INT}(A, B) & =1 / 2\{M E(B \mid A+)-M E(B \mid A-)\} \\
& =1 / 2\{M E(A \mid B+)-M E(A \mid B-)\} .
\end{aligned}
$$

Here, $M E(B \mid A+)$ is called the conditional effect of $B$ at the + level of $A$. The effect of one factor (e.g., $B$ ) therefore depends on the level of the other factor (e.g., A). Similar equations exist for higher order effects and for designs with nonbinary options. See $\mathrm{Wu}$ and Hamada [30] for further details.

Once the effects are computed, developers will want to determine which of them are important and which are not. There are several ways to determine this, including using standard hypothesis testing. We do not use formal hypothesis tests primarily because they require strong assumptions about the standard deviation of the experimental samples. Instead, we display the effects graphically and let developers use their expert judgment to decide which effects they consider important. While this approach has some downsides (see Section 4), even with traditional tests for statistical significance, experimenters must still judge for themselves whether a significant effect has any practical importance.

Our graphical analysis uses half-normal probability plots, which show each option's effect against their corresponding coordinates on the half-normal probability scale. If $|\theta|_{1} \leq$ $|\theta|_{2} \leq \ldots \leq|\theta|_{I}$ are the ordered set of effect estimations, the half-normal plot then consists of the points

$$
\left(\Phi^{-1}(0.5+0.5[i-0.5] / I),|\theta|_{i}\right) \text { for } i=1, \ldots, I,
$$

where $\Phi$ is the cumulative distribution function of a standard normal random variable.
The rationale behind half-normal plots is that unimportant options will have effects whose distribution is normal and centered near 0 . Important effects will also be normally distributed, but with means different from $0 .{ }^{3}$ Options whose effects deviate substantially from 0 should therefore be considered important. If no effects are important, the resulting plot will show a set of points on an approximate line near $y=0$.

\subsection{Conducting Supplementary Analysis}

At this point, developers have a candidate set of important options. One potential problem, however, is that we arrived at these options by making the following assumptions:

1. The low-order effects identified as being important really are, while the higher-order effects they are aliased to are not.

2. Monitoring only low-order effects is sufficient to produce reliable estimates.

Since these are only assumptions, it is important to check them before proceeding since the reliability of our results will be severely compromised if they do not hold. We therefore validate these assumptions using two types of follow-up experiments:

1. We first examine additional configurations to disambiguate any effects aliased to our purported lowlevel important options.

2. We then examine additional configurations to look for other higher-order effects.

In these follow-up experiments, we rely on another class of efficient experimental designs called D-optimal designs [22], which are, again, computer-aided designs. Given a configuration space and a model the experimenter wishes to fit, a D-optimal design uses search-based computer algorithms (e.g., hill climbing or simulated annealing) to select a set of configurations that satisfy a particular optimality criterion. Unlike more common fractional factorial designs, therefore, the size of D-optimal designs need not be a perfect fraction of full factorial designs. Doptimal designs are preferable to standard classical designs when 1) the standard designs require more observations than can be tested with available time and resources and 2) the configuration space is heavily constrained (i.e., when not all the configurations are valid). Both factors are frequently present in modern software systems. Full details of D-optimal designs are beyond the scope of this paper but can be found in books and articles (see Kolarik [11] and Mitchell [22]).

Based on the results of this D-optimal design analysis, developers may modify the set of important options. At this point, developers have a working set of important options that they can use to create a screening suite of configurations to benchmark whenever the system changes.

\section{FeasibILITy Study}

This section describes a feasibility study that assesses the implementation cost and the effectiveness of the reliable

3. Since the effects are averages over numerous observations, the central limit theorem guarantees normality. 
TABLE 2

Some ACE+TAO Options

\begin{tabular}{|c|c|c|c|}
\hline Option Index & Option Name & Option Settings & Option Description \\
\hline$\overline{\mathrm{A}}$ & ReactorThreadQueue & \{FIFO, LIFO $\}$ & $\begin{array}{l}\text { Order in which incoming requests } \\
\text { are processed in the ORB Reactor }\end{array}$ \\
\hline $\mathrm{B}$ & ClientConnectionHandler & $\{\mathrm{RW}, \mathrm{MT}\}$ & Client side connection handler \\
\hline $\mathrm{C}$ & ReactorMaskSignals & $\{0,1\}$ & $\begin{array}{l}\text { Enable/disable signals } \\
\text { during request processing }\end{array}$ \\
\hline $\mathrm{D}$ & ConnectionPurgingStrategy & $\{$ LRU, LFU $\}$ & ORB connection purging strategy \\
\hline $\mathrm{E}$ & ConnectionCachePurgePercent & $\{10,40\}$ & $\%$ of the ORB connection cache purged \\
\hline $\mathrm{F}$ & ConnectionCacheLock & $\{$ thread, null $\}$ & $\begin{array}{l}\text { Enable/disable locking of } \\
\text { the ORB connection cache }\end{array}$ \\
\hline G & CorbaObjectLock & $\{$ thread, null $\}$ & $\begin{array}{l}\text { Enable/disable locking while } \\
\text { synchronizing object state }\end{array}$ \\
\hline $\mathrm{H}$ & ObjectKeyTableLock & $\{$ thread, null $\}$ & $\begin{array}{l}\text { Type of lock to be used } \\
\text { within ORB for retrieving } \\
\text { object keys }\end{array}$ \\
\hline I & InputCDRAllocator & $\{$ thread, null $\}$ & $\begin{array}{l}\text { Enable/disable locking while } \\
\text { creating CDR streams }\end{array}$ \\
\hline $\mathbf{J}$ & Concurrency & $\begin{array}{l}\text { \{reactive, } \\
\text { thread-per-connect }\end{array}$ & $\begin{array}{l}\text { ORB concurrency } \\
\text { reactive or thread per connection }\end{array}$ \\
\hline $\mathrm{K}$ & ActiveObjectMapSize & $\{32,128\}$ & Map size for holding objects \\
\hline $\mathrm{L}$ & UseridPolicyDemuxStrategy & $\{$ linear, dynamic $\}$ & $\begin{array}{l}\text { Demultiplexing strategy } \\
\text { when user-id policy is used }\end{array}$ \\
\hline M & SystemPolicyDemuxStrategy & $\{$ linear, dynamic $\}$ & $\begin{array}{l}\text { Demultiplexing strategy } \\
\text { when system id policy is used }\end{array}$ \\
\hline $\mathrm{N}$ & UniquePolicyRevDemuxStrategy & $\{$ linear, dynamic $\}$ & $\begin{array}{l}\text { Specify the reverse demultiplexing } \\
\text { lookup strategy to be } \\
\text { used with the unique id policy }\end{array}$ \\
\hline
\end{tabular}

effects screening process described in Section 3 on a suite of large, performance-intensive software frameworks.

\subsection{Experimental Design}

Hypotheses. Our feasibility study explores the following three hypotheses:

1. Our MDE-based Skoll environment cost-effectively supports the definition, implementation, and execution of our reliable effects screening process described in Section 3.

2. The screening designs used in the reliable effects screening correctly identifies a small subset of options whose effect on performance is important.

3. Exhaustively examining just the options identified by the screening design gives performance data that 1) is representative of the system's performance across the entire configuration space, but less costly to obtain and 2) is more representative than a similarly sized random sample.

Subject applications. The experimental subject applications for this study were based on three open-source software frameworks for performance-intensive systems: ACE v5.4 + TAO v1.4 + CIAO v0.4, which can be downloaded via www.dre.vanderbilt.edu. ACE provides reusable $\mathrm{C}++$ wrapper facades and framework components that implements core concurrency and distribution patterns [26] for distributed real-time and embedded (DRE) systems. TAO is a highly configurable Real-time CORBA ORB built atop ACE to meet the demanding QoS requirements of DRE systems. CIAO extends TAO to support components, which enables developers to declaratively provision QoS policies end-to-end when assembling DRE systems.

$\mathrm{ACE}+\mathrm{TAO}+\mathrm{CIAO}$ are ideal subjects for our feasibility study since they share many characteristics with other highly configurable performance-intensive systems. For example, they collectively have more than 2 million lines of source code, functional regression tests, and performance benchmarks contained in $\sim 4,500$ files that average more than 300 CVS commits per week by dozens of developers around the world. They also run on a wide range of OS platforms, including all variants of Windows, most versions of UNIX, and many real-time operating systems, such as LynxOS and VxWorks.

Application scenario. Due to recent changes made to the $\mathrm{ACE}$ message queuing strategy, the developers of $\mathrm{ACE}+\mathrm{TAO}+\mathrm{CIAO}$ were concerned with measuring two performance criteria: 1) the latency for each request and 2) total message throughput (events/second) between the $\mathrm{ACE}+\mathrm{TAO}+\mathrm{CIAO}$ client and server. For this version of $\mathrm{ACE}+\mathrm{TAO}+\mathrm{CIAO}$, the developers identified 14 binary runtime options they felt affected latency and throughput. The entire configuration space therefore has $2^{14}=16,384$ different configurations. To save space, we refer to these options by their one letter indices, A-N (see Table 2 for more details on the mapping of letters to options).

Using MDE tools to model an experiment scenario. To ease experiment creation and generation, we used the Skoll MDE tools to compose the experiment visually. In particular, we used BGML to generate the platform-specific benchmarking code needed to evaluate the QoS of the ACE+TAO+CIAO software framework configurations. Fig. 3 shows how we used BGML to model the benchmark.

For the experiment, we modeled the operation exchanged between the client and server using BGML's operation element. We then associated this element with BGML's latency metric to compute the end-to-end measurements for our experiments. The number of warmup iterations and the data type exchanged between client and server were all set as attributes to the operation and latency elements provided by BGML. As shown in Fig. 3, BGML 


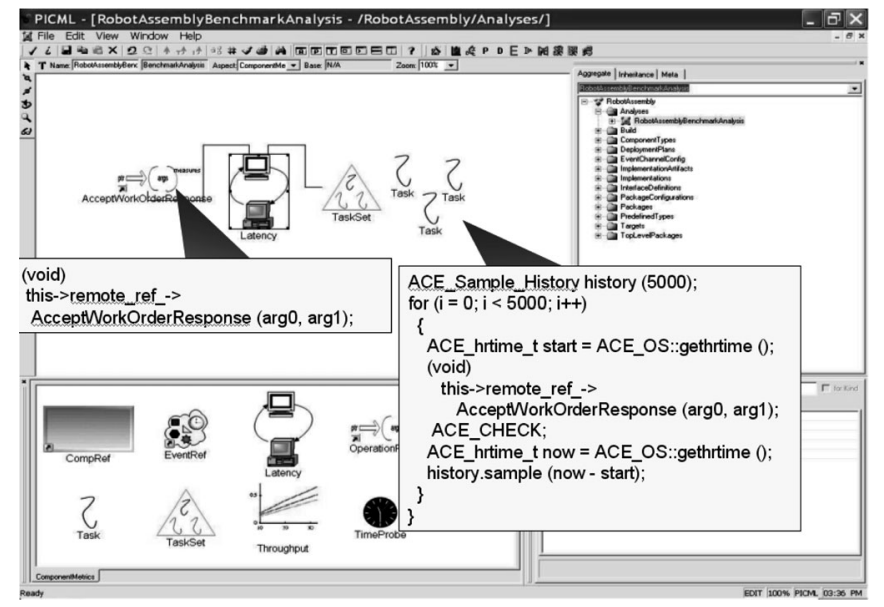

Fig. 3. Using BGML to generate benchmarking code.

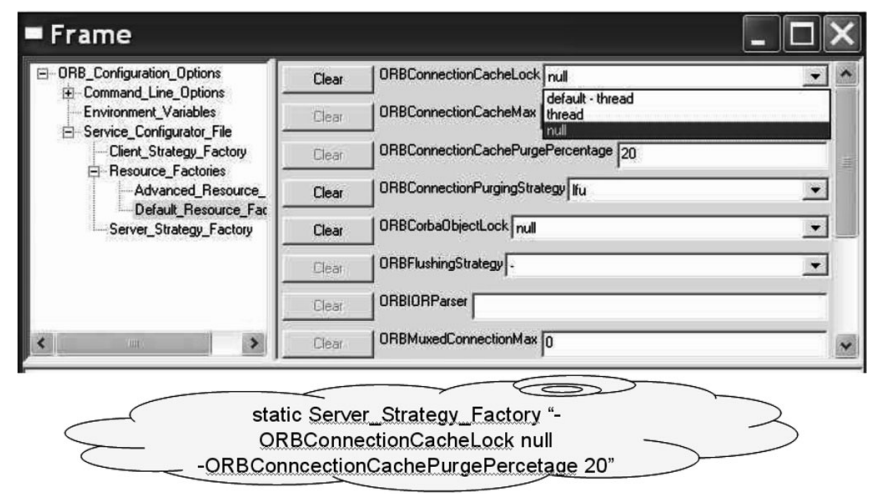

Fig. 4. Using OCML to capture experiment configuration.

code generators generated the benchmarking code to measure and capture the latency for our experiment.

Another step in designing our experiment involved modeling the $\mathrm{ACE}+\mathrm{TAO}+\mathrm{CIAO}$ framework configurations. We used the OCML MDE tool to ensure that the configuration were both syntactically and semantically valid, as shown in Fig. 4.

As shown in Fig. 4, OCML was used to enter the $\mathrm{ACE}+\mathrm{TAO}+\mathrm{CIAO}$ configurations we wanted to measure. OCML's constraint checker first validated the configurations we modeled, while the code generator produced the framework configuration files.

Experimental process. Our experimental process used Skoll's MDE tools to implement the reliable effects screening process and evaluate our three hypotheses above. We executed the reliable effects screening process across a prototype Skoll grid of dual processor Xeon machines running Red Hat 2.4.21 with 1 GB of memory in the realtime scheduling class. The experimental tasks involved running a benchmark application in a particular system configuration, which evaluated performance for the application scenario outlined above. The benchmark created an $\mathrm{ACE}+\mathrm{TAO}+\mathrm{CIAO}$ client and server and then measured message latency and overall throughput between the client and the server.

In our experiment, the client sent $300 \mathrm{~K}$ requests to the server. After each request the client waited for a response from the server and recorded the latency measure in microseconds. At the end of $300 \mathrm{~K}$ requests, the client

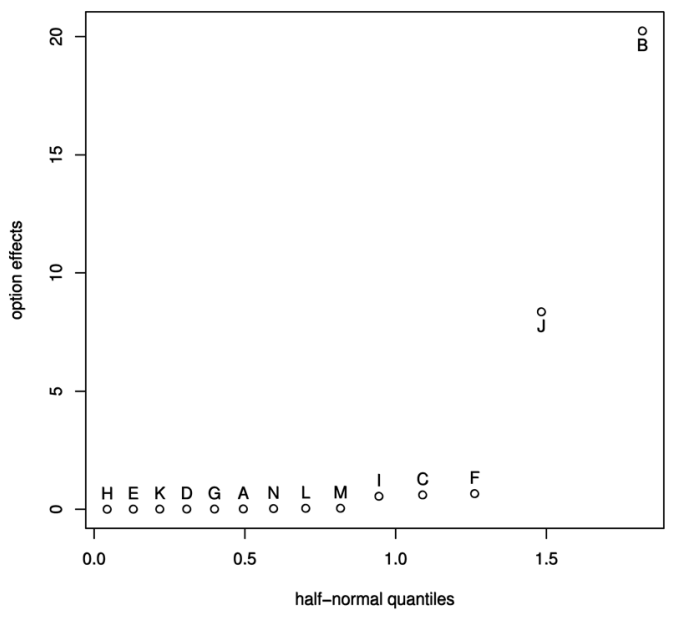

(a)

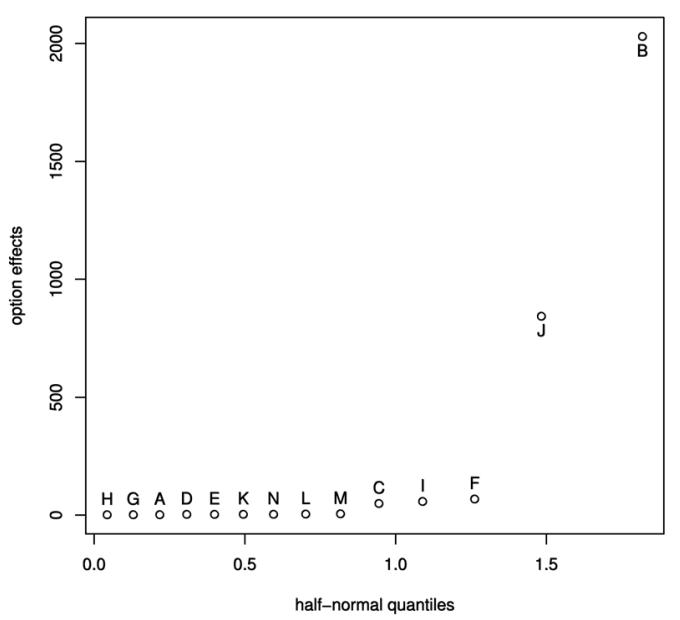

(b)

Fig. 5. Option effects based on full data. (a) Half-normal probability plot for latency (full-factorial). (b) Half-normal probability plot for throughput (full-factorial).

computed the throughput in terms of the number of requests served per second. We then analyzed the resulting data to evaluate our hypotheses. Section 8 describes the limitations with our current experimental process.

\subsection{The Full Data Set}

To provide a baseline for evaluating our approach, we first generated and analyzed performance data for all 16,000+ valid configurations of $\mathrm{ACE}+\mathrm{TAO}+\mathrm{CIAO}{ }^{4}$ We refer to these configurations as the "full suite" and the performance data as the "full data set."

We examined the effect of each option and judged whether they had important effects on performance. Fig. 5 plots the effect of each of the 14 ACE+TAO+CIAO options on latency and throughput across the full data set. We see that options $B$ and $J$ are clearly important, whereas options

4. We would not do this step in practice since it required about two days of CPU time, which would be prohibitively expensive in most production software development environments with scores of such experiments running daily. 


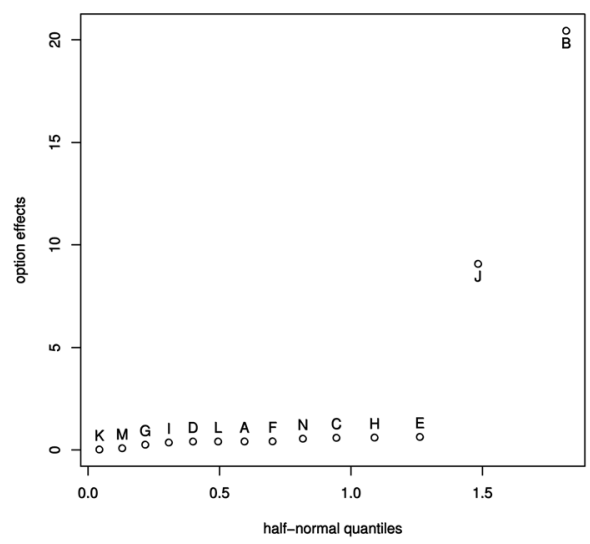

(a)

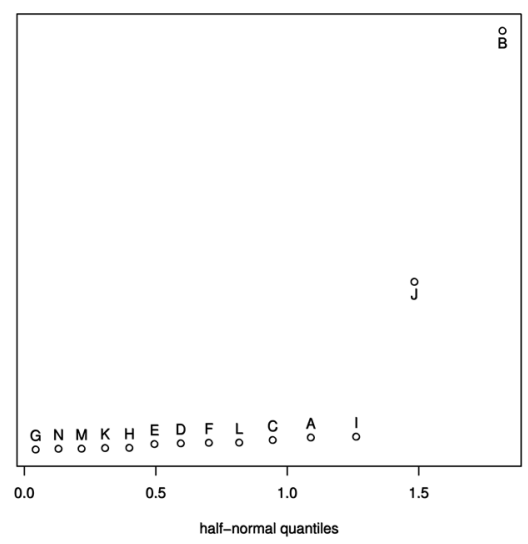

(b)

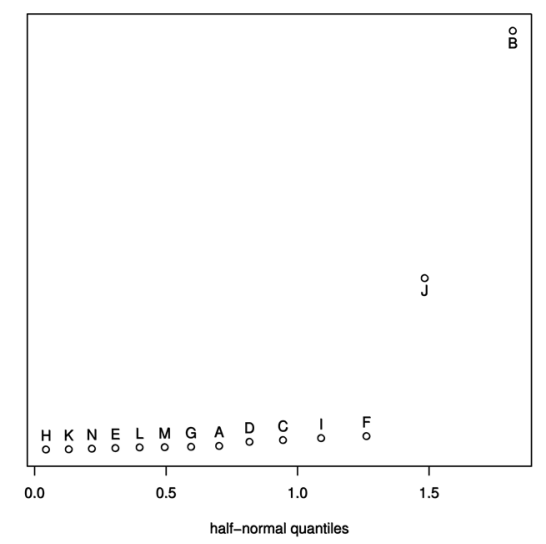

(c)

Fig. 6. (a) Half-normal probability plot for latency (resoultion IV, 32-run). (b) Half-normal probability plot for latency (resolution IV, 32-run). (c) Halfnormal probability plot for latency (resolution IV, 128-run).

I, $C$, and $F$ are arguably important. The remaining options are not important.

\subsection{Evaluating Screening Designs}

We now walk through the steps involved in conducting the reliable effects screening process to see whether the remotely executed screening designs can correctly identify the same important options discovered in the full data set.

To perform these steps, we calculated and executed several different resolution IV screening designs of differing run sizes. The specifications for these designs appear in the Appendix. The first set of designs examined all 14 options using increasingly larger run sizes $(32,64$, or 128 observations) to identify only important first-order effects. We refer to these screening designs as $S c_{32}, S c_{64}$, and $S r_{128}$, respectively. We also calculated and executed a second set of designs that attempted to capture important first- and second-order effects.

Fig. 6 shows the half-normal probability plots obtained from our first set of screening designs. The figures show

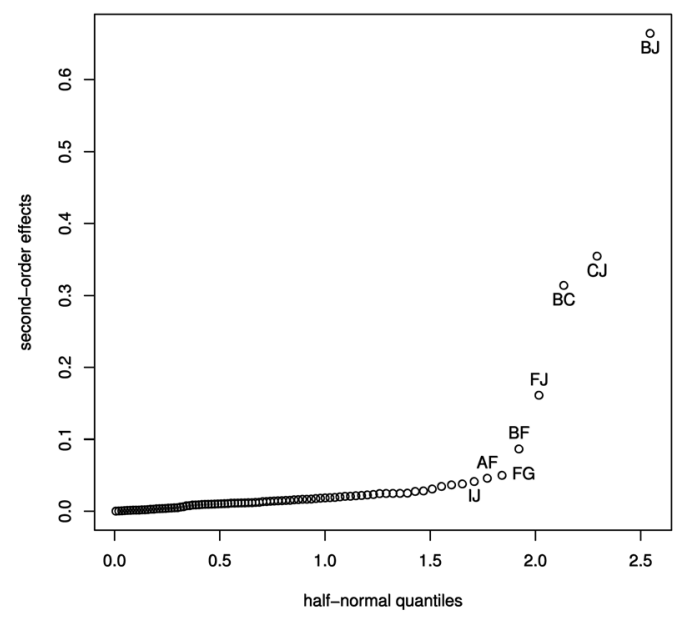

(a) that all screening designs correctly identify options $B$ and $J$ as being important. $\mathrm{Scr}_{128}$ also identifies the possibly important effect of options $C, I$, and $F$. Due to space considerations in the paper, we only present data on latency (throughput analysis showed identical results unless otherwise stated).

\subsection{Higher-Order Effects}

The resolution IV design only calculates clear first-order effects, which appears to work well for our subject application and scenario, but might not be sufficient for other situations. Fig. 7 shows the effects of all pairs of options based on the full data set and the same effects captured via a resolution VI screening design using 2,048 observations. From the figure we see several things: 1) the screening design correctly identifies the five most important pairwise interactions at one-eighth the cost of exhaustive testing and 2) the most important interaction effects involve only options that are already considered important by themselves, which supports the belief that

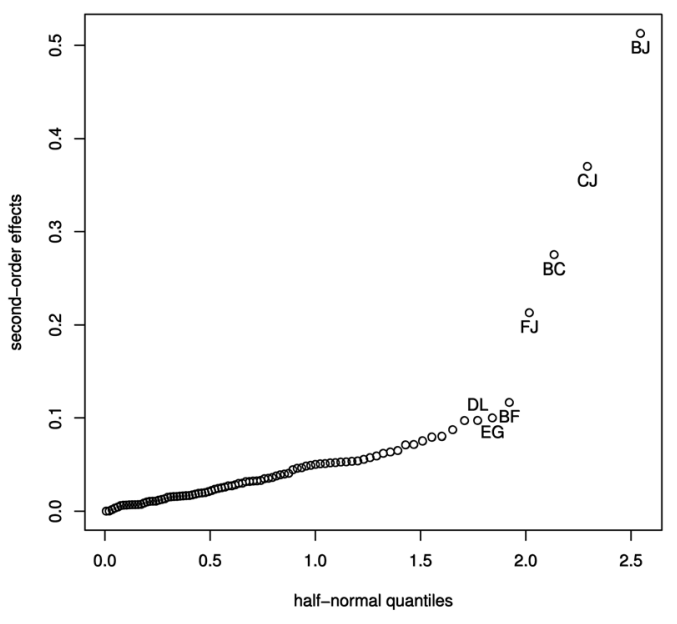

(b)

Fig. 7. Pairwise effects based on full and screening suites. (a) Half-normal probability plot for latency (full-factorial). (b) Half-normal probability plot for latency (resolution VI, 2,048-run). 
TABLE 3

Partial Aliasing Structure for the Important Option in the $2_{I V}^{14-7}$, 128-Run Design

Partial Aliasing Structure

$\mathrm{B}=\mathrm{ACH}=\mathrm{CGKM}=\mathrm{CKLN}=\mathrm{DGHL}=\mathrm{DHMN}=\mathrm{EFHK}=\mathrm{HIJK}=\mathrm{ADEI}=\mathrm{ADFJ}$

$\mathbf{J}=\mathrm{EFI}=\mathrm{ACIK}=\mathrm{BHIK}=\mathrm{CDFH}=\mathrm{DEGM}=\mathrm{DELN}=\mathrm{FGKN}=\mathrm{FKLM}=\mathrm{ABDF}$

$\mathrm{C}=\mathrm{ABH}=\mathrm{ADMN}=\mathrm{AIJK}=\mathrm{BGKM}=\mathrm{BKLN}=\mathrm{DEHI}=\mathrm{DFHJ}=\mathrm{AEFK}=\mathrm{ADGL}$

$\mathrm{F}=\mathrm{EIJ}=\mathrm{BEHK}=\mathrm{CDHJ}=\mathrm{DGIM}=\mathrm{DILN}=\mathrm{GJKN}=\mathrm{JKLM}=\mathrm{ABDJ}=\mathrm{ACEK}$

$\mathrm{I}=\mathrm{EFJ}=\mathrm{ACJK}=\mathrm{BHJK}=\mathrm{CDEH}=\mathrm{DFGM}=\mathrm{DFLN}=\mathrm{EGKN}=\mathrm{EKLM}=\mathrm{ABDE}$

monitoring only first-order effects will be sufficient for our subject frameworks.

\subsection{Validating Basic Assumptions}

To compute the important options, we used a resolution IV screening design, which according to the definition given in Section 3, means that 1) we aliased some first-order effects with some third-order or higher effects and some secondorder effects with other second-order or higher effects, and 2) we assume that third-order or higher effects are negligible. If these assumptions do not hold, however, performance estimations will be incorrect. Therefore, at this stage we perform two further analyses to validate these assumptions. In the remainder of this section, we analyze the data from our $2_{I V}^{14-7}, 128$-run experiment. We did this experiment because it was the only one where we identified five important options rather than two. The analysis presented in this section is readily applicable to any screening experiment.

\subsubsection{Breaking Aliases}

In the $2_{I V}^{14-7}, 128$-run experiment, we identified options $B$ and $J$ as being clearly important and options $C, F$, and $I$ as being arguably important (see Table 2 for the list of options). In that design, some first-order effects were aliased with some third-order or higher effects. Table 3 shows the aliasing structure for the important options up to fourth-order effects. Note that the complete aliasing structure for the important options contains a total of 640 aliases. Consider the alias $B=A C H$ for the important option $B$; the effect of option $B$ is aliased with the interaction effect of options $A, C$, and $H$. Since the experimental analysis cannot distinguish $B$ from $A C H$, there is an ambiguity whether $B$ or $A C H$ is really important. If $A C H$, not $B$, is the important effect, then our performance estimations would obviously suffer, as would the rest of the aliases. Ambiguities involving aliased effects for the important options should therefore be resolved to ensure the reliability of the performance estimations. To resolve the ambiguity in this experiment, we first formally modeled the important effects and their 640 aliases by the following linear formula:

$$
\begin{aligned}
y= & \beta_{0}+\beta_{B} x_{B}+\beta_{J} x_{J}+\beta_{C} x_{C}+\beta_{F} x_{F}+\beta_{I} x_{I} \\
& +\beta_{A C H} x_{A} x_{C} x_{H}+\beta_{i} x_{i}, \quad \forall i \in S-\{A C H\},
\end{aligned}
$$

where $S$ is the set of all 640 aliased effects, $\beta_{0}$ is the intercept term, $x_{B}=-1,1$ according to the level of option $\mathrm{B}$ (the definitions for the other $x \mathrm{~s}$ are similar), $\beta \mathrm{s}$ are the model coefficients, etc.

Note that since the first-order effects other than $B, J$, $C, F$, and $I$ are negligible (Section 4.3), we excluded them from the model. We then augmented our 128-run screening experiment using the D-optimal design approach. This search-based technique determined an appropriate design, which required benchmarking an additional 2,328 configurations. We then collected the results and analyzed them together with the results of the original screening experiments.

Fig. 8a plots the Type III sum of squares for the factorial effects. In this figure, the effects are ordered in descending order. Due to space limitations, only the top 10 effects are given. The higher the sum of squares, the more important the effects are.
Type III Sum of Squares for Effecta Screening

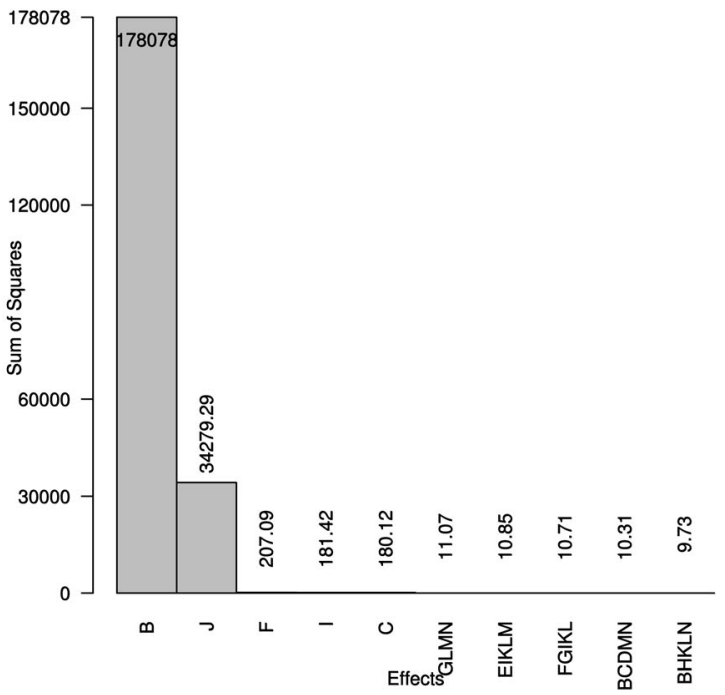

Type III Sum of Squares for Effect Screening

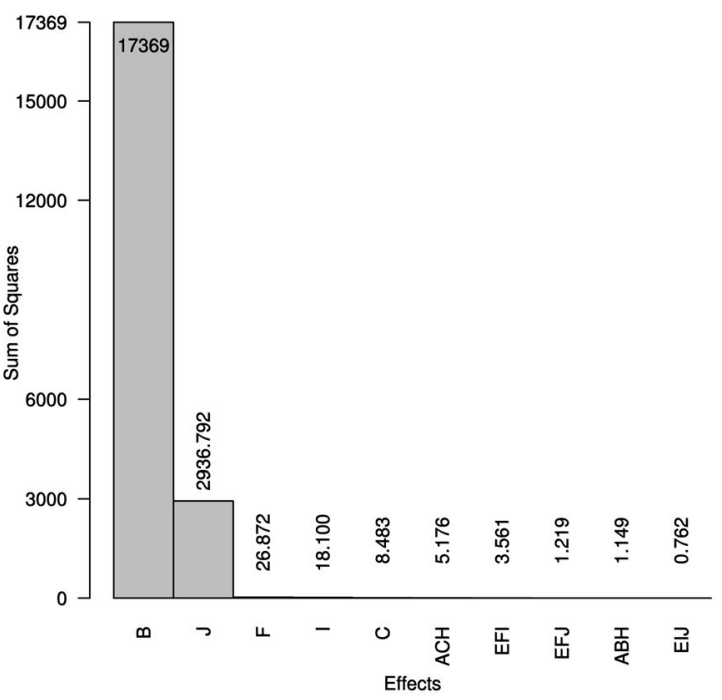

(a)

(b) 
As can be seen from this figure, options $B, J, C, F$, and $I$ are important, while the higher-order interactions to which they are aliased are not, i.e., the low-order effect explains roughly 10 times the variance of its higher-order alias. Further statistical analysis also showed that these options are statistically significant at 99.99 percent confidence level or better.

Although this analysis confirmed our earlier results, it required benchmarking an additional 2,000+ configuration, which in large-scale systems might too expensive. To further reduce costs, instead of a complete dealiasing of important options, one might consider dealiasing them up to a certain level of order (e.g., up to third-order or fourth-order effects) or dealiasing only suspected aliases based on developer's domain knowledge. To see how much savings partial dealiasing might yield, we repeated the evaluation process by dealiasing the important options only up to and including third-order effects. This required us to benchmark only an additional 64 configurations. As can be seen in Fig. 8b, in this particular example, we were able to reach the same conclusions as the complete dealiasing experiment.

\subsubsection{Checking for Higher-Order Effects}

As described earlier in Section 4.5, our second assumption was that monitoring only first-order effects was sufficient for our subject frameworks. Below, we investigate the validity of that assumption. Without loss of generality, we will look for important effects up to and including thirdorder effects. Developers can choose up to which level to examine, keeping in mind that as the level increases, the number of observations needed converges to exhaustive testing.

Just as in Section 4.5.1, we augmented the $2_{I V}^{14-7}, 128$-run experiment using the D-optimality criterion. This time, however, our generalized linear model consisted of all the first-order, second-order, and third-order effects. This design required only an additional 381 configurations. Fig. 9 shows the results of this study. We identified the top six effects: $B, J, F, I, B J$, and $C$, as statistically significant at a 99.9 percent confidence level or better. Among these important options, we have only one interaction effect: $B J$. Since this interaction involves only options that are already considered important by themselves, we conclude that monitoring only first-order effects was sufficient for our subject frameworks.

The results presented above suggest that screening designs can detect important options at a small fraction of the cost of exhaustive testing and that techniques exist to economically check key assumptions underlying the technique. The smaller the effect, however, the larger the run size needed to identify it. Developers should therefore be cautious when dealing with options that appear to have an important but relatively small effect, as they may actually be seeing normal variation $\left(S_{c r} r_{22}\right.$ and $S_{c r} r_{4}$ both have examples of this).

\subsection{Estimating Performance with Screening Suites}

Our experiments thus far have identified a small set of important options. We now evaluate whether benchmarking all combinations of these most important options can be
Type III Sum of Squares for Effect Screening

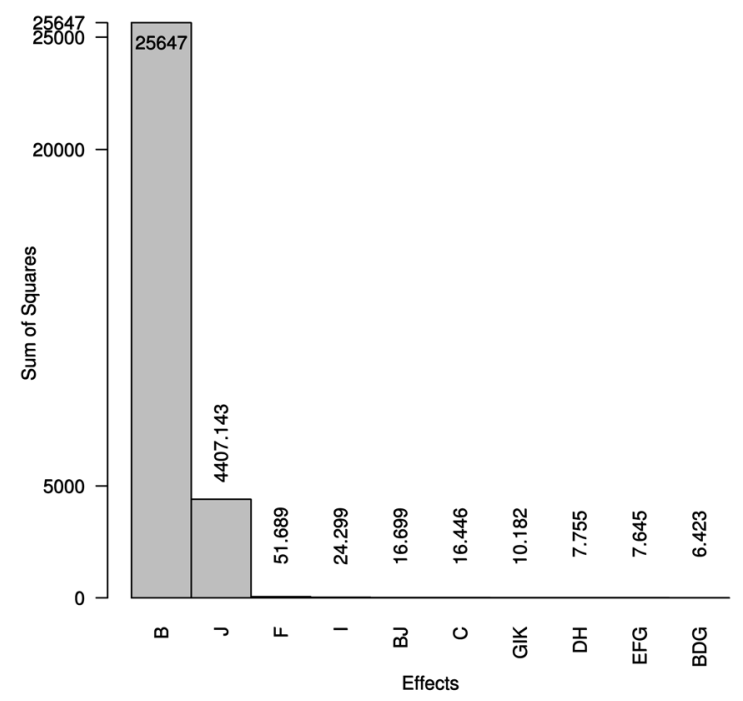

Fig. 9. Looking for higher-order effects up to and including third-order effects.

used to estimate performance quickly across the entire configuration space we are studying. The estimates are generated by examining all combinations of the most important options while randomizing the settings of the unimportant options.

In Section 4.3, we determined that options $B$ and $J$ were clearly important and that options $C, I$, and $F$ were arguably important. Developers therefore made the estimates based on benchmarking either 4 (all combinations of options $B$ and $J$ ) or 32 (all combinations of options $B, J, C, I$, and $F$ ) configurations. We refer to the set of 4 configurations as the "top-2 screening suite" and the set of 32 configurations as the "top-5 screening suite."

Fig. 10 shows the distributions of latency for the full suite versus the top-2 screening suite and for the full suite versus the top- 5 screening suite. The distributions of the top- 2 and top-5 screening suites closely track the overall performance data. Such plots, called quantile-quantile (Q-Q) plots, are used to see how well two data distributions correlate by plotting the quantiles of the first data set against the quantiles of the second data set. If the two sets share the same distribution, the points should fall approximately on the $x=y$ line.

We also performed Mann-Whitney non-parametric tests [3] to determine whether each set of screening data (top-2 and top-5 suites) appears to come from the same distribution as the full data. In both cases, we were unable to reject the null hypothesis that the top- 2 and top- 5 screening suite data come from the same distribution as the full suite data. These results suggest that the screening suites computed at Step 4 of the reliable effects screening process (Section 3) can be used to estimate overall performance in-house at extremely low time/effort, i.e., running 4 benchmarks takes 40 seconds, running 32 takes 5 minutes, and running 16,000+ takes two days of CPU time. 


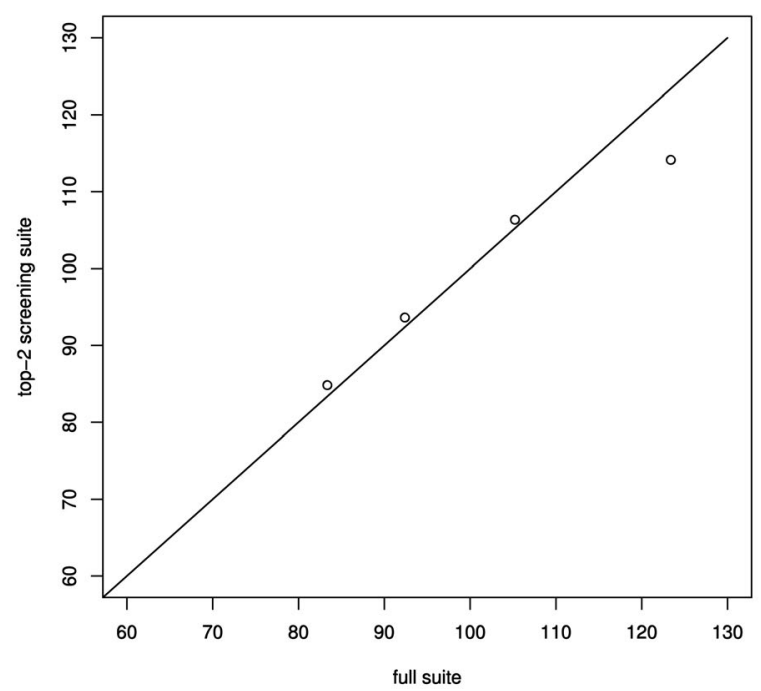

Fig. 10. Q-Q plots for latency for the top-2 and top-5 screening suites.

\subsection{Screening Suites versus Random Sampling}

Another question we addressed is whether our reliable effects screening process was better than other low-cost estimation processes. In particular, we compared the latency distributions of several random samples of four configurations to that of the top-2 screening suite found by our process. The results of this test are summarized in Fig. 11. These box plots show the distributions of latency metric obtained from exhaustive testing, top-2 screening suite testing, and random testing. These graphs suggest the obvious weakness of random sampling, i.e., while sampling distributions tend toward the overall distribution as the sample size grows, individual small samples may show wildly different distributions.

\subsection{Dealing with Evolving Systems}

The primary goal of reliable effects screening is to detect performance degradations in evolving systems quickly. So far, we have not addressed whether-or for how long-

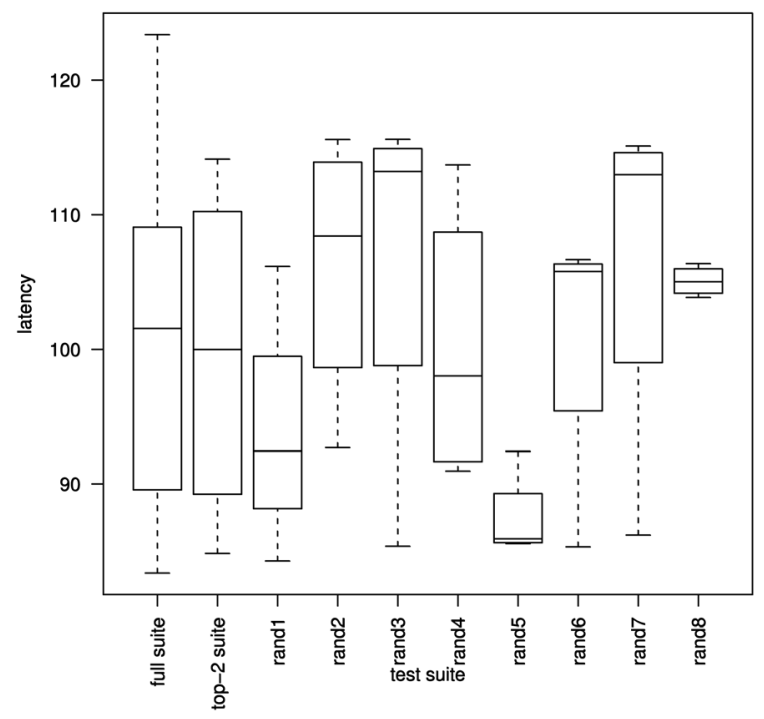

Fig. 11. Latency distributions from full, top-2, and random suites.

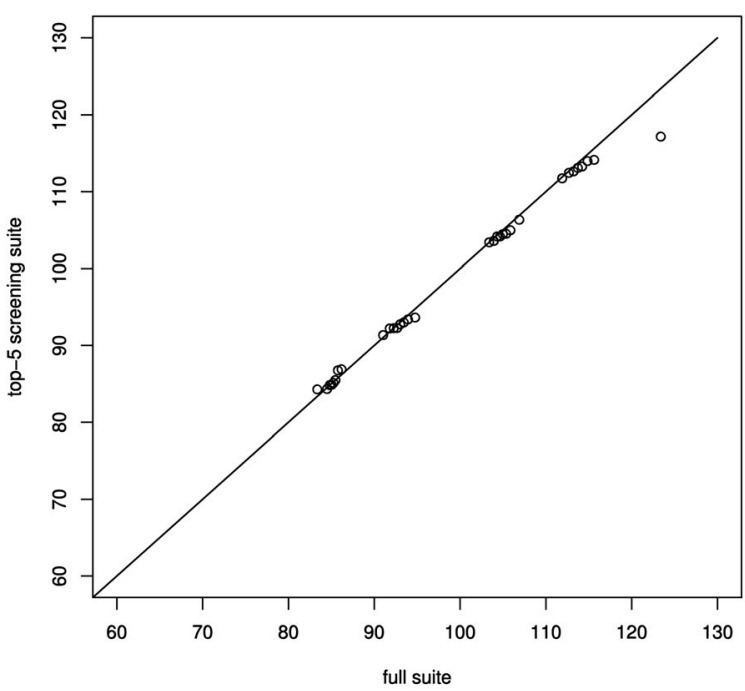

screening suites remain useful as a system evolves. To better understand this issue, we measured latency on the top-2 screening suite, once a day, using CVS snapshots of ACE+TAO+CIAO. We used historical snapshots for two reasons: 1) the versions are from the time period for which we already calculated the effects and 2) developer testing and in-the-field usage data have already been collected and analyzed for this time period (see www.dre.vanderbilt. edu/Stats), allowing us to assess the system's performance without having to test all configurations for each system change exhaustively.

Fig. 12 depicts the data distributions for the top-2 screening suites broken down by date (higher latency measures are worse).

We see that the distributions were stable the first two days, crept up somewhat for days 3 through 5 , and then shot up day 6 (14 December 2003). They were brought back under control for several more days, but then moved up again on the last day. Developer records and problem

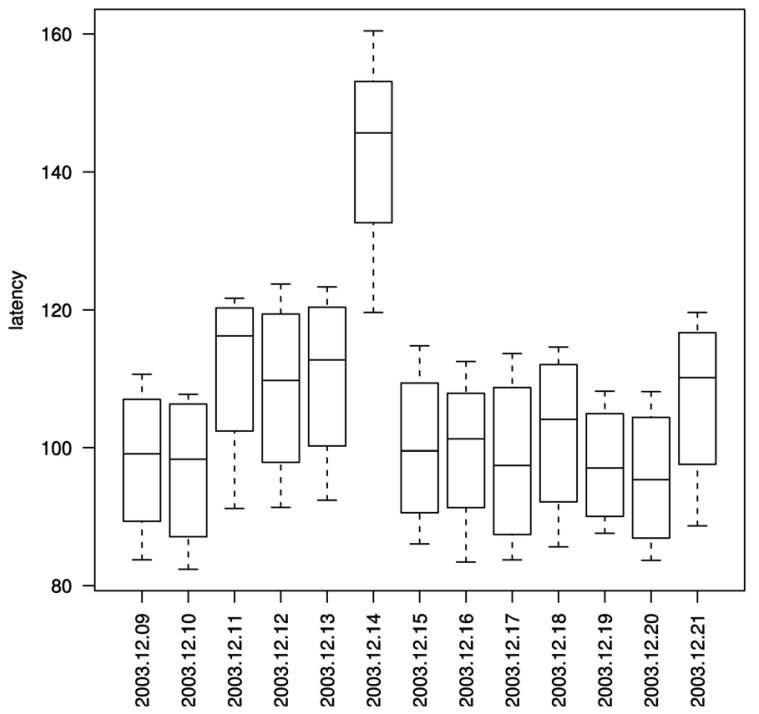

Fig. 12. Performance estimates across time. 
reports indicate that problems were not noticed until 14 December 2003.

Another interesting finding was that the limited in-house testing conducted by the $\mathrm{ACE}+\mathrm{TAO}+\mathrm{CIAO}$ developers measured a performance drop of only around 5 percent on 14 December 2003. In contrast, our screening process showed a much more dramatic drop-closer to 50 percent. Further analysis by ACE+TAO+CIAO developers showed that their unsystematic testing failed to evaluate configurations where the degradation was much more pronounced.

Taken together, our results suggest that benchmarking only all combinations of the important options identified in steps 1 through 4 of the reliable effects screening process gives much the same information as benchmarking the entire configuration space, but at a substantially reduced cost.

\section{Discussion OF FEASIBILITY StUdY}

\subsection{Threats to Validity}

All empirical studies suffer from threats to their internal and external validity. For the experiments described in Section 4, we were primarily concerned with threats to external validity since they limit our ability to generalize the results of our experiment to industrial practice. One potential threat is that several steps in our process require human decision making and input. For example, developers must provide reasonable benchmarking applications and must also decide which effects they consider important.

Another possible threat to external validity concerns the representativeness of the $\mathrm{ACE}+\mathrm{TAO}+\mathrm{CIAO}$ subject applications, which are an integrated suite of software (albeit a very large suite with more than 2 million lines of code). A related issue is that we have focused on the subset of the entire configuration space of $\mathrm{ACE}+\mathrm{TAO}+\mathrm{CIAO}$ that only has binary options and has no interoption constraints. While these issues pose no theoretical problems (screening designs can be created for much more complex situations), there is clearly a need to apply reliable effects screening to larger and richer configuration spaces in future work to understand how well the process scales.

Another potential threat is that for the time period we studied, the $\mathrm{ACE}+\mathrm{TAO}+\mathrm{CIAO}$ subject frameworks were in a fairly stable phase. In particular, changes were made mostly to fix bugs and reduce memory footprint, but the software's core functionality was relatively stable. For time periods where the core software functionality is in greater flux, e.g., in response to new requirements from sponsors or efforts to port the software to new and different platforms, it may be harder to distinguish significant performance degradation from normal variation.

\subsection{Hypotheses}

Despite the limitations described in Section 5.1, we believe the study presented in Section 4 supports our basic hypotheses presented in Section 4.1. We reached this conclusion by noting that our study suggests that the 1) MDE-based Skoll system allows $Q A$ engineers to quickly construct complex DCQA processes, 2) reliable effects screening process provides developers with fast, cheap and reliable estimates of performance across a system's entire configuration space, and 3) developers of
TABLE 4

Generated Code Summary for BGML

\begin{tabular}{|cccc|}
\hline Files & Number & Lines of Code & Generated (\%) \\
\hline \hline IDL & 3 & 81 & 100 \\
Source (.cpp) & 2 & 310 & 100 \\
Header (.h) & 1 & 108 & 100 \\
Script (.pl) & 1 & 115 & 100 \\
\hline
\end{tabular}

ACE+TAO+CIAO believe the technique provides them with important development information.

Benefits from applying our MDE tools. Our MDE tools helped improve the productivity of QA engineers by allowing them to create QA task models and to compose benchmarking experiments visually rather than wrestling with low-level formats and source code. These tools thus resolve tedious and error-prone accidental complexities associated with writing correct code by autogenerating them from higher level models. For example, Table 4 summarizes the BGML code generation metrics for a particular configuration.

This table shows how BGML automatically generates 8 of 10 required files that account for 88 percent of the code required for the experiment. Since these files must capture specific information for each configuration, these tools imply large improvements in productivity for performing benchmarking QA tasks. Similarly, OCML enabled us to generate both syntactically and semantically correct middleware configurations, thereby eliminating accidental complexity in generating middleware configurations.

Reliable effects screening. Our experiments showed that the reliable effects screening process was fast, cheap, and effective. We came to this conclusion by noting that:

1. Screening designs can correctly identify important options (Section 4.3, Section 4.4, and Section 4.5).

2. These options can be used to produce reliable estimates of performance quickly across the entire configuration space at a fraction of the cost of exhaustive testing (Section 4.6).

3. The alternative approach of random or ad hoc sampling can give highly unreliable results (Section 4.7).

4. The reliable effects screening process detected performance degradation on a large and evolving software system (Section 4.8.).

5. The screening suite estimates were significantly more precise than the ad hoc process currently used by the developers of ACE+TAO+CIAO (Section 4.8).

User acceptance. Informally, we found that ACE+ TAO+CIAO developers have been quite happy with the results of our experiments described in Section 4. As we move toward fully integrating reliable effects screening into their development processes, they continue to find new ways in which this information can help them improve their development processes, including:

Using option importance to prioritize work. Our quantitative results showed that options $L, M$, and $N$ did not have a strong effect on latency and throughput. These findings surprised some ACE+TAO+CIAO developers, who had spent considerable time optimizing code affected by these options. Further investigation showed that the 
options can have a somewhat larger effect, but only in very specific circumstances. The developers now see reliable effects screening as a means to better understand how widespread the effects of different pending changes may be.

Using changes in option importance to detect bugs. Prior to the release of an ACE+TAO+CIAO beta, developers noted a significant $(\sim 25$ percent $)$ drop in performance. Since reliable effects screening had not yet been fully integrated into their development processes, the ACE+TAO+CIAO developers fell back on traditional ad hoc QA and debugging techniques. When they failed to identify the problem's cause, they froze their CVS repository and used an exhaustive QA approach to painstakingly narrow down the change that had degraded performance.

Ultimately, they found the change that caused the degradation was a feature addition that enabled TAO+CIAO to support the IPv6 protocol. Specifically, they observed that a modification to TAO's connection handler had degraded performance. Interestingly, this code is controlled by option $B$. In retrospect, a reliable effects screening would have shown a dramatic change in the importance of option $B$, thus enabling the ACE+TAO+CIAO developers to localize the problem quickly, i.e., because one setting of option $B$ triggered the buggy code, option $B^{\prime}$ s effect would have been 2.5 times greater after the change than before it. Based on this experience, the developers now see added value in frequently recalibrating the important options to alert them to changes in option importance.

\section{UsAge GuideLINES}

This section focuses on providing some guidelines on how to use the Skoll-based reliable effects screening process. We examine how to select an appropriate resolution and size for the screening designs, how to identify the important options, and how to validate the basic assumptions in identifying the important options. We also summarize on our experience in applying this process on the ACE+TAO+CIAO software frameworks.

Step 1: Choose the resolution. Leverage a priori knowledge of the software being tested, if it is available, to decide the resolution of the screening experiment, i.e., which highorder effects are considered important versus negligible. If no or limited a priori information is available, use screening experiments in an iterative manner (e.g., going from lower resolutions to higher ones) to obtain this information. Section 4.1 illustrated how we did this for the ACE+TAO+ CIAO software frameworks, where we selected 14 specific options to explore based on prior knowledge of a recent change.

Step 2: Choose the size. Depending on available resources and how fast the underlying software changes, determine the maximum number of observations allowed in the screening experiment. Note that the resolution of the experiment chosen in Step 1 may dictate the minimum size of the experiment. If this size is not feasible, consider lowering the resolution of the experiment by carefully choosing the aliasing structure so that no potentially important higher-order effects are aliased with lower-order ones. Sections 4.3 and 4.4 illustrate how we did this for the $\mathrm{ACE}+\mathrm{TAO}+\mathrm{CIAO}$ software frameworks, where we created three resolution IV designs with run sizes of 32, 64, and 128, and where we created one resolution VI design with a run size of 2,048.

Step 3: Identify the important options. After the screening design is computed, conducted, and analyzed, use the half-normal probability plots described in Section 4.3 to identify important options. If no effects are important, these plots will show a set of points on a rough line near $y=0$. Any substantial deviations from this line indicate important options. Depending on the benchmarking test and the desired precision of the performance estimates, decide how large effects must be to warrant attention. Section 4.3 shows how we did this for the ACE+TAO+CIAO software frameworks, where we identified two important and three arguably important options.

Step 4: Validate the basic assumptions. If needed, validate the assumptions imposed by the choice of the resolution. Use D-optimal designs described in Section 4.5 to augment the screening experiment to 1) dealias the important options and 2) identify remaining higher-order effects. Section 4.5 illustrated how we did this for the ACE+TAO+CIAO software frameworks and showed that our basic assumptions helped and that our initial analysis was therefore reliable.

Step 5: Estimate performance after changing software. Focusing on important options allows developers to reduce the effective configuration space significantly by evaluating all combinations of the important options, while randomizing the rest. Section 4.5 illustrated how we did this for the ACE+TAO+CIAO software frameworks and showed that our approach gave reliable estimates of performance across the entire configuration space using only 40 seconds (for top-2 suite) or 2 minutes (for top-5 suite) of CPU time.

Step 6: Frequently recalibrate important options. The importance of different options may change as software in a system changes. We therefore recommend frequent recalibration of the important effects. Although our feasibility study in Section 4 does not show the need for recalibration, our experience applying reliable effects screening to $\mathrm{ACE}+\mathrm{TAO}+\mathrm{CIAO}$ over time indicates that recalibration is essential.

\section{Related Work}

This section compares our work on reliable effects screening and performance evaluation techniques in Skoll with other related research efforts, including

1. applying design-of-experiments (DOE) testing to software engineering,

2. large-scale testbed environments for conducting experiments using heterogeneous hardware, OS, and compiler platforms,

3. evaluating the performance of layered software systems,

4. feedback-based optimization techniques that use empirical data and mathematical models to identify performance bottlenecks.

Applying DOE to software engineering. As far as we know, we are the first to use screening designs to assess software performance. The use of DOE theory within 
software engineering has focused mostly on interaction testing, largely to compute and sometimes to generate minimal test suites that cover all combinations of specified program inputs. Mandl [19] first used orthogonal arrays, a special type of covering array in which all $t$-sets occur exactly once, to test enumerated types in ADA compiler software. This idea was extended by Brownlie et al. [5], who developed the orthogonal array testing system (OATS). They provided empirical results to suggest that the use of orthogonal arrays is effective in fault detection and provides good code coverage.

Dalal et al. [8] argue that the testing of all pairwise interactions in a software system finds a large percentage of the existing faults. In further work, Burr and Young [6], Dunietz et al. [9], and Kuhn and Reilly et al. [15] provide more empirical results to show that this type of test coverage is effective. These studies focus on finding unknown faults in already tested systems and equate covering arrays with code coverage metrics. Yilmaz et al. [31] used covering arrays as a configuration space sampling technique to support the characterization of failure-inducing option settings.

Large-scale benchmarking testbeds. EMULab [29] is a testbed at the University of Utah that provides an environment for experimental evaluation of networked systems. EMULab provides tools that researchers can use to configure the topology of their experiments, e.g., by modeling the underlying OS, hardware, and communication links. This topology is then mapped to $\sim 250$ physical nodes that can be accessed via the Internet [24]. The EMULab tools can generate script files that use the Network Simulator (NS) (www.isi.edu/nsnam/ns) syntax and semantics to run the experiment.

The Skoll infrastructure provides a superset of EMULab that is not limited by resources of a particular testbed but, instead, can leverage the vast end-user computer resources in the Skoll grid. Moreover, the Skoll's MDE-based tools described in Section 2 can generate NS scripts to integrate our benchmarks with experiments in EMULab.

Feedback-driven optimization techniques. Traditional feedback-driven optimization techniques can be divided into online, offline, and hybrid analysis. Offline analysis has commonly been applied to program analysis to improve compiler-generated code. For example, the ATLAS [34] numerical algebra library uses an empirical optimization engine to decide the values of optimization parameters by generating different program versions that are run on various hardware/OS platforms. The output from these runs are used to select parameter values that provide the best performance. Mathematical models are also used to estimate optimization parameters based on the underlying architecture, though empirical data is not fed into the models to refine it.

Like ATLAS, Skoll's MDE tools use an optimization engine to configure/customize software parameters in accordance to available OS platform characteristics (such as the type of threading, synchronization, and demultiplexing mechanisms) and characteristics of the underlying hardware (such as the type of CPU, amount of main memory, and size of cache). This information can be used to select optimal configurations ahead of time that maximize
QoS behavior. Online analysis is commonly used for feedback control to adapt QoS behaviors based on dynamic measures. An example of online analysis is the ControlWare middleware [35], which uses feedback control theory by analyzing the architecture and modeling it as a feedback control loop. Actuators and sensors then monitor the system and affect server resource allocation. Real-time scheduling based on feedback loops has also been applied to Real-time CORBA middleware [18] to automatically adjust the rate of remote operation invocation transparently to an application.

Though online analysis enables systems to adapt at runtime, the optimal set of QoS features are not determined at system initialization. Using the MDE tools, QoS behavior and performance bottlenecks on various hardware and software configurations can be determined offline and then fed into the models to generate optimal QoS characteristics at model construction time. Moreover, dynamic adaptation can incur considerable overhead from system monitoring and adaptation, which may be unacceptable for performance-intensive DRE systems. Hybrid analysis combines aspects of offline and online analysis. For example, the continuous compilation strategy [7] constantly monitors and improves application code using code optimization techniques. These optimizations are applied in four phases including

1. static analysis, in which information from training runs is used to estimate and predict optimization plans,

2. dynamic optimization, in which monitors apply code transformations at run-time to adapt program behavior,

3. offline adaptation, in which optimization plans are actually improved using actual execution, and

4. recompilation, where the optimization plans are regenerated.

Skoll's MDE-based strategy enhances conventional hybrid analysis by tabulating platform-specific and platformindependent information separately using the Skoll framework. In particular, Skoll does not incur the overhead of system monitoring since behavior does not change at runtime. New platform-specific information obtained can be fed back into the models to optimize QoS measures.

Generative Benchmarking Techniques. There have been several initiatives that use generative techniques similar to our approach for generating test-cases and benchmarking for performance evaluation. The ForeSight [17] tool uses empirical benchmarking engine to capture QoS information for COTS based component middleware system. The results are used to build mathematical models to predict performance. This is achieved using a threepronged approach of 1) create a performance profile of how components in a middleware affect performance, 2) construct a reasoning framework to understand architectural trade-offs, i.e., know how different QoS attributes interact with one another and 3) feed this configuration information into generic performance models to predict the configuration settings required to maximize performance.

The SoftArch/MTE [10] tool provides a framework for system architects to provide higher-level abstraction of the 
system specifying system characteristics such as middleware, database technology, and client requests. The tool then generates an implementation of the system along with the performance tests that measure system characteristics. These results are then displayed back, i.e., annotated in the high-level diagrams, using tools such as Microsoft Excel, which allows architects to refine the design for system deployment.

Our MDE approach closely relates to the aforementioned approaches. Both the ForeSight and SoftArch tools, however, lack DCQA environments to help capture QoS variations accurately on a range of varied hardware, OS, and compiler platforms. Rather than using generic mathematical models to predict performance, MDE tools use a feedback-driven approach [13], wherein the DCQA environment is used to empirically evaluate the QoS characteristics offline. This information can then be used to provide QA engineers with accurate system information. Moreover, platform-specific and application-specific optimization techniques [12] can then be applied to maximize QoS characteristics of the system.

\section{Concluding Remarks}

This paper presents a new distributed continuous quality assurance (DCQA) process called "reliable effects screening" that uses in-house and in-the-field resources to efficiently and reliably detect performance degradation in performance-intensive systems that have large configuration spaces. The novelty of our approach stems from its application of statistical quality control techniques to efficiently identify a subset of system configurations that accurately represents the performance of the entire configuration space. Benchmarking this subset after a change provides a reliable estimate of the distribution of performance across all configurations. Unexpected changes in this distribution signal unintended side effects that must be examined further.

To evaluate the reliable effects screening process, we integrated it with the Skoll DCQA environment and applied it to three large software projects (ACE, TAO, and CIAO) using a grid of computing resources managed by the Skoll DCQA environment. Skoll's reliable effects screening process is also supported by model-driven engineering (MDE) tools that control and manage its execution across a grid of in-house and in-the-field computing resources. The results of our experiments in this environment indicated that:

- The reliable effects screening process helped developers detect and understand performance bottlenecks across large configuration spaces.

- The ACE+TAO+CIAO developers used the information provided by this process as a scalable defect detection aid, i.e., when the important options change unexpectedly (at recalibration time), developers reexamined the frameworks to rapidly identify possible problems with software updates.

- $\mathrm{ACE}+\mathrm{TAO}+\mathrm{CIAO}$ developers also used information provided by the reliable effects screening process to understand and arbitrate disputes about subtle changes in framework and application performance characteristics.

In conclusion, we believe that this line of research is novel and fruitful, though much R\&D remains to be done. We are therefore continuing to develop enhanced MDEbased Skoll capabilities and use them to create and validate more sophisticated DCQA processes that overcome the limitations and threats to external validity described in Section 5. In particular, we are exploring the connection between design-of-experiments theory and the QA of other software systems with large software configuration spaces. We are also incorporating the tools in the Skoll environment and the reliable effects screening process into open-source software repositories, such as ESCHER (www.escherinstitute.org). Finally, we are conducting a much larger case study using Skoll and reliable effect screening to orchestrate the ACE+TAO+CIAO daily build and regression test process with more than 200 machines contributed by users and developers worldwide, as shown on our online DCQA scoreboard at www.dre.vanderbilt. edu/scoreboard.

\section{APPENDIX}

The screening designs used in Section 4.3 were calculated using the SAS statistical package (www.sas.com).

$S_{C r} r_{32}$ is a $2_{I V}^{14-9}$ with design generators $F=A B C$, $G=A B D, \quad H=A C D, \quad I=B C D, \quad J=A B E, \quad K=A C E$, $L=B C E, M=A D E, N=B D E$.

$\mathrm{Scr}_{64}$ is a $2_{I V}^{14-8}$ with design generators $G=A B C$, $H=A B D, I=A B E, J=A C D E, K=A B F, L=A C D F$, $M=A C E F, N=A D E F$.

$S c r_{128}$ is a $2_{I V}^{14-7}$ with design generators $H=A B C$, $I=A B D E, \quad J=A B D F, \quad K=A C E F, L=A C D G, \quad M=$ $A B E F G, N=B C D E F G$.

The screening designs used in Section 4.4 were calculated using the SAS statistical package (www.sas.com).

They are $2_{V I}^{14-3}$ (2,048-run) designs with design generators $L=A B C D E F G H I J K, M=E F G H I J K$, and $N=$ $C D G H I J K$.

\section{ACKNOWLEDGMENTS}

This work was done when Balachandran Natarajan was with the Department of Electrical Engineering and Computer Science at Venderbilt University. This material is based on work supported by the US National Science Foundation under NSF grants ITR CCR-0312859, CCF0447864, CCR-0205265, and CCR-0098158, as well as funding from BBN, Cisco, the US Defense Advanced Research Projects Agency, Lockheed Martin Advanced Technology Lab and Advanced Technology Center, ONR, Qualcomm, Raytheon, and Siemens. The authors thank the anonymous reviewers for their comments that helped improve this paper.

\section{REFERENCES}

[1] SAS Institute, www. sas. com/.

[2] K. Balasubramanian, A. Gokhale, G. Karsai, J. Sztipanovits, and S. Neema, "Developing Applications Using Model-Driven Design Environments," Computer, vol. 39, no. 2, pp. 33-40 2006. 
[3] G.E.P. Box, W.G. Hunter, and J.S. Hunter, Statistics for Experimenters, John Wiley \& Sons, 1978.

[4] L. Breiman, J. Freidman, R. Olshen, and C. Stone, Classification and Regression Trees. Wadsworth, 1984.

[5] R. Brownlie, J. Prowse, and M.S. Padke, "Robust Testing of AT\&T PMX/StarMAIL using OATS," ATET Technical J., vol. 71, no. 3, pp. 41-47, 1992.

[6] K. Burr and W. Young, "Combinatorial Test Techniques: TableBased Automation, Test Generation and Code Coverage," Proc. Int'l Conf. Software Testing Analysis E Rev., 1998.

[7] B. Childers, J. Davidson, and M. Soffa, "Continuous Compilation: A New Approach to Aggressive and Adaptive Code Transformation," Proc. Int'l Parallel and Distributed Processing Symp., Apr. 2003.

[8] S.R. Dalal, A. Jain, N. Karunanithi, J.M. Leaton, C.M. Lott, G.C. Patton, and B.M. Horowitz, "Model-Based Testing in Practice," Proc. Int'l Conf. Software Eng. (ICSE), pp. 285-294, 1999.

[9] I.S. Dunietz, W.K. Ehrlich, B.D. Szablak, C.L. Mallows, and A. Iannino, "Applying Design of Experiments to Software Testing," Proc. Int'l Conf. Software Eng. (ICSE '97), pp. 205-215, 1997.

[10] J. Grundy, Y. Cai, and A. Liu, "Generation of Distributed System Test-beds from High-level Software Architecture Description," 16th Int'l Conf. Automated Software Eng., Sept. 2001.

[11] W. Kolarik, Creating Quality: Systems, Concepts, Strategies and Tools. McGraw-Hill Education, 1995

[12] A.S. Krishna, A. Gokhale, D.C. Schmidt, V.P. Ranganath, and J. Hatcliff, "Model-Driven Middleware Specialization Techniques for Software Product-Line Architectures in Distributed Real-time and Embedded Systems," Proc. MODELS Workshop MDD for Software Product-Lines, Oct. 2005.

[13] A.S. Krishna, D.C. Schmidt, A. Porter, A. Memon, and D. SevillaRuiz, "Improving the Quality of Performance-intensive Software via Model-integrated Distributed Continuous Quality Assurance," Proc. Eighth Int'l Conf. Software Reuse, 2004.

[14] A.S. Krishna, N. Wang, B. Natarajan, A. Gokhale, D.C. Schmidt, and G. Thaker, "CCMPerf: A Benchmarking Tool for CORBA Component Model Implementations," Proc. 10th Real-Time Technology and Application Symp. (RTAS '04), May 2004.

[15] D. Kuhn and M. Reilly, "An Investigation of the Applicability of Design of Experiments to Software Testing," Proc. 27th Ann. NASA Goddard/IEEE Software Eng. Workshop, pp. 91-95, 2002.

[16] A. Ledeczi, A. Bakay, M. Maroti, P. Volgysei, G. Nordstrom, J. Sprinkle, and G. Karsai, "Composing Domain-Specific Design Environments," Computer pp. 44-51, Nov. 2001.

[17] Y. Liu, I. Gorton, A. Liu, N. Jiang, and S. Chen, "Designing a Test Suite for Empirically-Based Middleware Performance Prediction," Proc. 40th Int'l Conf. Technology of Object-Oriented Languages and Systems, Aug. 2002.

[18] C. Lu, J.A. Stankovic, G. Tao, and S.H. Son, "Feedback Control Real-Time Scheduling: Framework, Modeling, and Algorithms," Real-Time Systems J., vol. 23, nos. 1-2, pp. 85-126, July 2002.

[19] R. Mandl, "Orthogonal Latin Squares: An Application of Experiment Design to Compiler Testing," Comm. ACM, vol. 28, no. 10, pp. 1054-1058, 1985.

[20] A. Memon, A. Porter, C. Yilmaz, A. Nagarajan, D.C. Schmidt, and B. Natarajan, "Skoll: Distributed Continuous Quality Assurance," Proc. 26th IEEE/ACM Int'l Conf. Software Eng., May 2004.

[21] A.M. Memon, M.E. Pollack, and M.L. Soffa, "Hierarchical GUI Test Case Generation Using Automated Planning," IEEE Trans. Software Eng., vol. 27, no. 2, pp. 144-155, Feb. 2001.

[22] T. Mitchell, "An Algorithm for the Construction of the 'DOptimal' Experimental Designs," Technometrics, vol. 16, no. 2, pp. 203-210, 1974.

[23] D.C. Montgomery, Introduction to Statistical Quality Control, third ed. John Wiley \& Sons, 1996.

[24] R. Ricci, C. Alfred, and J. Lepreau, "A Solver for the Network Testbed Mapping Problem," SIGCOMM Computer Comm. Rev., vol. 33, no. 2, pp. 30-44, Apr. 2003.

[25] D.C. Schmidt, "Model-Driven Engineering," Computer, vol. 39, no. 2, pp. 25-31, 2006

[26] D.C. Schmidt, M. Stal, H. Rohnert, and F. Buschmann, PatternOriented Software Architecture: Patterns for Concurrent and Networked Objects, vol. 2. John Wiley \& Sons, 2000.

[27] J.W. Tukey, Exploratory Data Analysis. Addison-Wesley, 1977.

[28] E. Turkay, A. Gokhale, and B. Natarajan, "Addressing the Middleware Configuration Challenges Using Model-Based Techniques," Proc. 42nd Ann. Southeast Conf., Apr. 2004.
[29] B. White et al., "An Integrated Experimental Environment for Distributed Systems and Networks," Proc. Fifth Symp. Operating Systems Design and Implementation, pp. 255-270, Dec. 2002.

[30] C.F.J. Wu and M. Hamada, Experiments: Planning, Analysis, and Parameter Desion Optimization. Wiley, 2000.

[31] C. Yilmaz, M. Cohen, and A. Porter, "Covering Arrays for Efficient Fault Characterization in Complex Configuration Spaces, Proc. Int'l Symp. Software Testing and Analysis (ISSTA), pp. 45-54, 2004.

[32] C. Yilmaz, M.B. Cohen, and A. Porter, "Covering Arrays for Efficient Fault Characterization in Complex Configuration Spaces," IEEE Trans. Software Eng., vol. 31, no. 1 pp. 20-34, Jan. 2006.

[33] C. Yilmaz, A.S. Krishna, A. Memon, A. Porter, D.C. Schmidt, A Gokhale, and B. Natarajan, "Main Effects Screening: A Distributed Continuous Quality Assurance Process for Monitoring Performance Degradation in Evolving Software Systems," Proc. 27th Int' Conf. Software Eng. (ICSE '05), pp. 293-302, 2005.

[34] K. Yotov, X. Li, G. Ren, M. Cibulskis, G. DeJong, M. Garzaran, D. Padua, K. Pingali, P. Stodghill, and P. Wu, "A Comparison of Empirical and Model-Driven Optimization," Proc. ACM SIGPLAN Conf. Programming Language Design and Implementation, June 2003.

[35] R. Zhang, C. Lu, T. Abdelzaher, and J. Stankovic, "Controlware: A Middleware Architecture for Feedback Control of Software Performance," Proc. Int'l Conf. Distributed Systems, July 2002.

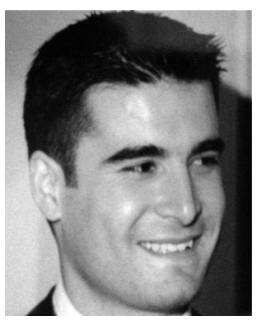

Cemal Yilmaz received the BS and MS degrees in computer engineering and Information Science from Bilkent University, Ankara, Turkey, in 1997 and 1999, respectively. In 2002 and 2005, he received the MS and PhD degrees in computer science from the University of Maryland at College Park. He is currently a postdoctoral researcher at the IBM Thomas $\mathrm{J}$. Watson Research Center, Hawthorne, New York, where he works in the field of software quality assurance. His current research interests include automated fault localization, performance modeling and optimization, distributed and adaptive quality assurance, formal methods in testing, and highly configurable systems. Dr. Yilmaz is a member of the ACM and the IEEE Computer Society.

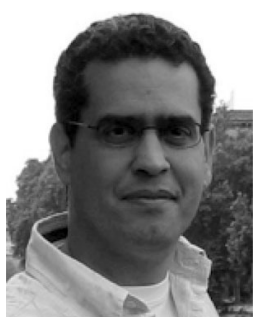

Adam Porter received the BS degree summa cum laude in computer science from the California State University at Dominguez Hills, Carson, California, in 1986. In 1988 and 1991, he received the MS and $\mathrm{PhD}$ degrees from the University of California at Irvine. Currently an associate professor, he has been with the Department of Computer Science and the Institute for Advanced Computer Studies at the University of Maryland since 1991. He is a winner of the US National Science Foundation Faculty Early Career Development Award and the Dean's Award for Teaching Excellence in the College of Computer, Mathematics, and Physical Sciences. His current research interests include empirical methods for identifying and eliminating bottlenecks in industrial development processes, experimental evaluation of fundamental software engineering hypotheses, and development of tools that demonstrably improve the software development process. Dr. Porter is a senior member of the ACM and the IEEE.

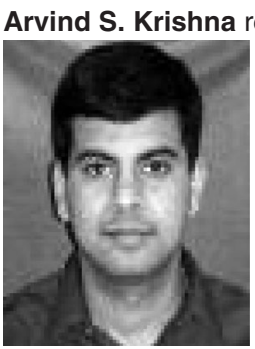

eived the masters and $\mathrm{PhD}$ degrees in computer science from the University of California at Irvine and Vanderbilt University. He is a senior engineer at Qualcomm, where he is currently working on wireless broadband networks. Before joining Qualcomm, He worked on building distributed object computing middleware and techniques for middleware customization. His interests include design patterns, design of flexible and customizable middleware, and model-driven development techniques. 


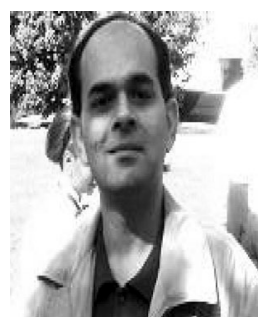

Atif $\mathrm{M}$. Memon received the $\mathrm{BS}, \mathrm{MS}$, and $\mathrm{PhD}$ degrees in computer science in 1991, 1995, and 2001, respectively. He is an assistant professor at the Department of Computer Science, University of Maryland. He was awarded a Gold Medal in his undergraduate studies. He was awarded Fellowships from the Andrew Mellon Foundation for his $\mathrm{PhD}$ research. He received the US National Science Foundation CAREER award in 2005. His research interests include program testing, software engineering, artificial intelligence, plan generation, reverse engineering, and program structures. He is a member of the ACM and the IEEE Computer Society.

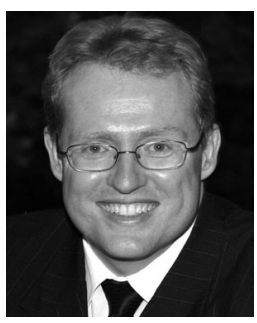

Douglas C. Schmidt is a professor of computer science and an associate chair of the Computer Science and Engineering Program at Vanderbilt University. He has authored more than 300 technical papers and eight books that cover a range of research topics, including patterns, optimization techniques, and empirical analyses of software frameworks and domain-specific modeling environments that facilitate the development of distributed real-time and embedded (DRE) middleware and applications running over high-speed networks and embedded system interconnects.

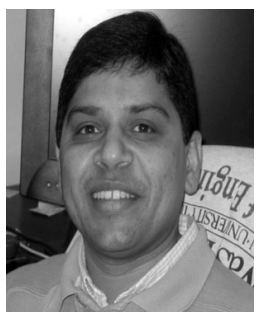

Aniruddha S. Gokhale received the bachelor of engineering degree in computer engineering from the University of Pune, India in 1989, the master of science degree in computer science from Arizona State University, Tempe, in 1992, and the doctor of science degee from Washington University, St. Louis, Missouri, in 1998. He is an assistant professor in the Electrical Engineering and Computer Science Department and a senior research scientist at the Institute for Software Integrated Systems (ISIS), both at Vanderbilt University, Nashville, Tennessee. Dr. Gokhale's research interests are in real-time component middleware optimizations, model-driven engineering applied to component middleware-based applications, and distributed resource management. Dr. Gokhale is heading the R\&D efforts on an open source model driven engineering framework called CoSMIC (www.dre. vanderbilt.edu/cosmic). He is a member of the ACM and the IEEE Communications Society.

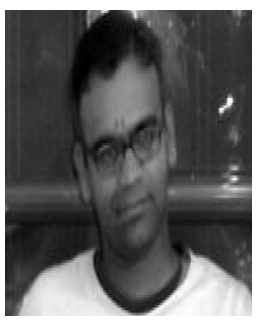

Balachandran Natarajan received the MS degree in computer science from Washington University in St. Louis, Missouri. He is a senior principal software engineer at Symantech, India. Prior to his current position, he was a senior staff engineer with the Institute for Software Integrated Systems at Vanderbilt University, Nashville, Tennessee. He has also worked as a software consultant developing software and tools for CAD, CAM, and CAE applications.

$\triangleright$ For more information on this or any other computing topic, please visit our Digital Library at www.computer.org/publications/dlib. 